\title{
A Comprehensive Three-Phase Step Voltage Regulator Model with Efficient Implementation in the Z-Bus Power Flow
}

This paper was downloaded from TechRxiv (https://www.techrxiv.org).

\section{LICENSE}

CC BY 4.0

SUBMISSION DATE / POSTED DATE

05-07-2020 / 14-07-2021

\section{CITATION}

Pompodakis, Evangelos; Kryonidis, Georgios C.; Alexiadis, Minas (2020): A Comprehensive Three-Phase Step Voltage Regulator Model with Efficient Implementation in the Z-Bus Power Flow. TechRxiv. Preprint. https://doi.org/10.36227/techrxiv.12612434.v3

DOI

10.36227/techrxiv.12612434.v3 


\title{
A Comprehensive Three-Phase Step Voltage Regulator Model with Efficient Implementation in the Z-Bus Power Flow
}

\author{
Evangelos E. Pompodakis, Georgios C. Kryonidis, Member, IEEE, and Minas C. Alexiadis \\ Evangelos E. Pompodakis* , Georgios C. Kryonidis ${ }^{\dagger}$, and Minas C. Alexiadis ${ }^{\ddagger}$ \\ ${ }^{*}{ }^{*}$ Department of Electrical and Computer Engineering, Aristotle University of Thessaloniki, Greece \\ Email: ${ }^{*}$ bobodakis@ hotmail.com, ${ }^{\dagger}$ kryonidi@ece.auth.gr, ${ }^{\dagger}$ minalex@ auth.com
}

\begin{abstract}
This paper presents a comprehensive three-bus equivalent circuit model of three-phase step voltage regulators. The proposed model can be efficiently integrated in the $Z$-bus power flow method and can accurately simulate any configuration of step voltage regulators. In contrast to the conventional step voltage regulator models that include the tap variables inside the $\mathbf{Y}_{\mathrm{BuS}}$ matrix

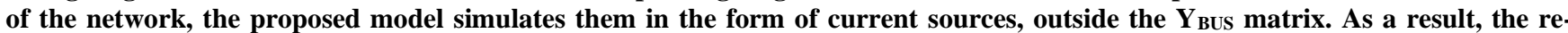
factorization of the $\mathbf{Y}_{\text {BUS }}$ matrix is avoided after every tap change reducing significantly the computational burden of the power flow. Furthermore, possible convergence issues caused by the low impedance of step voltage regulators are addressed by introducing fictitious impedances, without, however, affecting the accuracy of the model. The results of the proposed step voltage regulator model are compared against well-known commercial softwares such as Simulink and OpenDSS using the IEEE 4-Bus and an 8-Bus network. According to the simulations, the proposed model outputs almost identical results with Simulink and OpenDSS confirming its high accuracy. Furthermore, the proposed 3-bus equivalent model is compared against a recently published conventional step voltage regulator model in the IEEE 8500-Node test feeder. Simulation results indicate that the proposed step voltage regulator model produces as accurate results as the conventional one, while its computation time is significantly lower. More specifically, in the large IEEE 8500-node network consisting of four SVRs, the proposed model can reduce the computation time of power flow around one minute for every tap variation. Therefore, the proposed step voltage regulator model can constitute an efficient simulation tool in applications where subsequent tap variations are required.
\end{abstract}

Index Terms-Autotransformer, compensation technique, implicit $\mathrm{Z}_{\mathrm{BU}}$ power flow, step voltage regulator, tap variations, $Y_{\mathrm{BU}}$ matrix factorization.

List of Acronyms and Abbreviations

$\begin{array}{ll}\text { Step Voltage Regulator } & \text { SVR } \\ \text { Low Voltage } & \text { LV } \\ \text { Medium Voltage } & \text { MV } \\ \text { Backward Forward Sweep } & \text { BFS } \\ \text { Newton Raphson } & \text { NR } \\ \text { On Load Tap Changer } & \text { OLTC } \\ \text { Local Voltage Controller } & \text { LVC } \\ \text { Distribution Management System } & \text { DMS } \\ \text { Volt Var Control } & \text { VVC } \\ \text { Optimal Feeder Reconfiguration } & \text { OFR } \\ \text { Optimal Power Flow } & \text { OPF } \\ \text { Distributed Generator } & \text { DG }\end{array}$

\section{Introduction}

S TEP voltage regulators (SVRs) are widely applied to N low- (LV) and medium-voltage (MV) distribution networks to maintain voltages within permissible limits. They consist of autotransformers with adjustable turn ratios connected in several configurations e.g open-delta, closedelta, wye [1].

\subsection{Literature Review on the steady-state SVR modeling}

Only a few models have been proposed in the literature so far for the mathematical integration of SVRs into the power flow [1]-[4]. More specifically, the authors in [2] and [3] propose a mathematical formulation relating the primary and secondary voltages and currents, which is solved using the backward/forward sweep (BFS) method. In [4], a mathematical model of SVR is proposed, which is directly integrated in the Jacobian matrix, while the power flow is solved using the Newton-Raphson (NR) method.

However, these approaches are not applicable to the Z-Bus power flow since the SVR equations of [2]-[4] are not compatible with the formation of $\boldsymbol{Y}_{\boldsymbol{B} U S}$ matrix. The Z-Bus power flow approach is a fixed-point iterative method, which is widely applied in distribution network applications, due to its high robustness, fast convergence, ease of implementation and low computation time [5]-[9]. Therefore, accurate and computationally efficient modelling of SVRs is significant in order for the Z-Bus method to maintain its superior characteristics.

Considering the integration of SVR models in the threephase implicit Z-Bus power flow, only a limited number of solutions has been proposed in the literature. A comprehensive SVR model is proposed in [1], which is applicable in all SVR configurations, while an open-delta SVR model is proposed in [10]. Although the aforementioned models are applicable in the Z-Bus power flow approach, the tap variables of SVR are simulated in the $\boldsymbol{Y}_{\boldsymbol{B} U \boldsymbol{S}}$ matrix of the network. As a result, a re-factorization (or inversion) of the $Y_{\text {BUS }}$ matrix is required after every tap variation, thus increasing significantly the overall computational burden.

\subsection{Challenges of efficiently modeling SVRs in the Z-Bus power flow}

Ideally, the tap variables of the SVRs should be simulated outside the $\mathrm{Y}_{\mathrm{BUS}}$ matrix of the network so that the refactorization of the $\mathrm{Y}_{\mathrm{BUS}}$ matrix is performed offline, only once, accelerating the power flow calculation. The 
compensation technique is usually implemented in the $\mathrm{Z}_{\mathrm{BUS}}$ power flow to avoid the re-factorization of the $\boldsymbol{Y}_{\boldsymbol{B U S}}$ matrix every time that one or more elements change [11]. According to this technique, the variable elements are modeled as fictitious current sources outside the $\boldsymbol{Y}_{\boldsymbol{B} \text { US }}$ matrix, thus the $\boldsymbol{Y}_{\boldsymbol{B} U \boldsymbol{S}}$ matrix remains always constant.

The compensation technique is applied in [7] and [11] to simulate transformers equipped with on-load tap changer (OLTC) in balanced networks (using single-phase power flow) and by the authors in [12] to simulate three-phase OLTC transformers in $Y_{g}-Y_{g}$ configuration. However, in case of the SVRs, the implementation of the compensation technique results in the divergence of the power flow, due to the small impedance of SVRs, which in turn increases unacceptably the fictitious current sources. Similar conclusions about the divergence issues associated with the modeling of SVRs are derived in [13].

\subsection{Technical contribution of this paper}

This paper proposes a novel model, which overcomes the aforementioned divergence issues. The proposed model consists of a 3-bus equivalent circuit, which presents the following distinct characteristics:

- It simulates the taps of SVR as fictitious current sources outside the $\boldsymbol{Y}_{\text {BUS }}$ matrix, thus avoiding its continuous refactorization after every tap variation. As a result, several real-time distribution management system (DMS) applications that require sequential tap variations are accelerated. Simulations conducted in the IEEE 8500node network indicate that the proposed SVR model can reduce considerably the computation time of the power flow compared with the SVR model of [1].

- It is generic and can be applied in all SVR configurations i.e., wye, open-delta, closed-delta and types of SVRs i.e., Type A and Type B.

- The proposed model presents accurate power flow results since the applied equations are derived considering the exact three-phase SVR circuit. Simulation results confirm that the proposed model outputs identical power flow results with OpenDSS, Simulink and the model of [1], but with a significantly lower computation time.

- Although the taps are simulated in the form of current sources, the convergence of power flow is not compromised. Simulation results of the IEEE 8500-node network indicate that the proposed SVR model presents fast convergence speed.

\subsection{Applicability of the proposed model}

A modern DMS includes several real-time optimization applications such as Volt/Var control (VVC) [20] [21], optimal feeder reconfiguration (OFR) and optimal power flow (OPF) [7], which are executed following the load variations and the variation of the volatile distributed generator (DG) power [22] [23]. More specifically, as soon as an estimation of the system load and the volatile DG power is obtained, DMS optimization functions compute new switching states of the local voltage controllers (e.g SVRs,
OLTCs, capacitor banks, distributed generators) to optimize the system performance. The real-time power flow serves as an internal tool in the optimization functions since it is employed to simulate the effect of control actions on the state of the system and determines, if a new switching state of local voltage controllers (LVCs) is to be implemented [11]. Therefore, accurate and computationally efficient integration of LVCs in the power flow is essential for the efficient implementation of DMS optimization functions. The proposed SVR model can constitute an important tool in DMS applications, due to its high accuracy and its ability to maintain the structure of $\mathrm{Y}_{\mathrm{BUS}}$ matrix constant saving significant computation time.

Another important application of DMS is the state estimation and monitoring of the distribution system. It is realized through the execution of power flow considering the different time delays of the LVCs of the network. More specifically, LVCs do not react instantaneously but they are assigned to react with different reaction delays [18] [19]. For instance, an SVR placed near the substation may react faster after a voltage violation occurs than an SVR placed in a farther distance. Moreover, LVCs are assigned different voltage bandwidths, inside which the LVC state remains unchanged. Depending on the reaction sequence of LVCs, the power flow could yield multiple solutions, satisfying the voltage constraints. Therefore, in order to find the most probable power flow solution in networks with LVCs, the actual reaction sequence of the LVCs should be considered based on their time delays, voltage settings and bandwidths. This process requires the execution of power flow many times by subsequently varying the taps of LVCs based on their reaction time delays, until the final state is reached [11] [18] [19] [35]. With the existing SVR models, a high amount of computation time would be required, due to the continuous refactorization of $\mathrm{Y}_{\mathrm{BUS}}$ matrix every time that a tap variation occurs. This limitation is completely addressed with the proposed SVR model.

\subsection{Paper structure}

The rest of the paper is structured as follows: Section 2 explains the difference between the impedance value of an autotransformer and a two-winding transformer. Section 3 presents the proposed SVR model, while Section 4 explains how it is integrated in the Z-Bus power flow. Section 5 validates the proposed SVR model against Simulink and OpenDSS. Section 6 presents an analytical case study, in which the performance of the proposed SVR model is highlighted in the IEEE 8500-network. Finally, section 7 concludes the paper.

\section{Impedance of autotransformers}

Before we proceed with the analysis of the proposed SVR model, it is important to clarify the difference between a two separate winding transformer and an autotransformer. Fig. 1 depicts an ideal two-winding transformer and an ideal autotransformer. The impedances of the transformers are denoted as $Z_{2 w T}$ and $Z_{\text {auto }}$ and they include the total leakage impedance of the primary and secondary winding referred to 
the secondary winding. The primary voltage $\left(V_{1}\right)$, the secondary voltage $\left(V_{2}\right)$ and the number of turns of the primary windings $\left(N_{1}\right)$ are identical for both transformers.
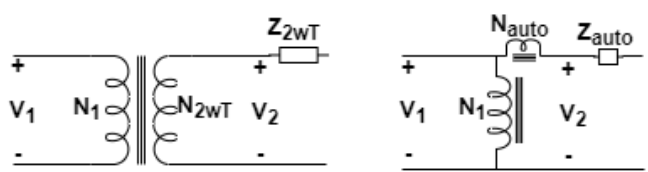

Fig. 1. Two-winding transformer (left) and autotransformer (right).

Voltages and turn numbers for the two types of transformers can be determined according to (1) and (2).

$$
\begin{gathered}
\frac{V_{2}}{V_{1}}=\frac{N_{2 w T}}{N_{1}} \Longrightarrow N_{2 w T}=\frac{V_{2}}{V_{1}} \cdot N_{1} \\
\frac{V_{2}}{V_{1}}=\frac{N_{\text {auto }}+N_{1}}{N_{1}} \Rightarrow N_{\text {auto }}=\frac{V_{2}}{V_{1}} \cdot N_{1}-N_{1}
\end{gathered}
$$

It can be observed that the number of turns of secondary windings $\left(N_{2 w T}\right.$ and $\left.N_{\text {auto }}\right)$ differs for the two transformer types. Furthermore, assuming that the two transformers have an equal magnetic reluctance, the corresponding impedance ratio for the two transformer types is given by (3).

$$
\frac{Z_{\text {auto }}}{Z_{2 w T}}=\left(\frac{N_{\text {auto }}}{N_{2 w T}}\right)^{2}
$$

By combining (1)-(3), (4) is derived, which gives the impedance ratio of the two types of transformer, as a function of the output/input voltage ratio $\left(V_{2} / V_{1}\right)$.

$$
\frac{Z_{\text {auto }}}{Z_{2 w T}}=\left(\frac{\frac{V_{2}}{V_{1}}-1}{\frac{V_{2}}{V_{1}}}\right)^{2}
$$

Fig. 2 depicts the impedance ratio of the two transformer types with respect to the output/input voltage ratio. The figure is obtained using (4) for an output/input voltage ratio between 0.9 and 1.1, which is a typical voltage range for SVRs. It can be observed that the autotransformer presents significantly lower impedance than the two-winding transformer. As a result of the low impedance of the autotransformer, the $Z_{\mathrm{BUS}}$ power flow diverges when the compensation technique is applied to the SVR modeling [13].

\section{Proposed SVR model}

Fig. 3 shows an SVR of Type A in closed-delta connection. The SVR is connected between buses $p$ and $s . Z_{\text {auto }}$ represents the autotransformer impedance of each phase. To overcome the divergence issues resulted from the low impedance of $Z_{\text {auto }}$, an additional fictitious bus $m$ is added in the 2-Bus circuit of Fig. 3, forming the 3-Bus circuit depicted at the top of Fig. 4. Moreover, an impedance $\left(Z_{a d d}\right)$ is added in series with $Z_{\text {auto }}$ so that $Z_{\text {pmi }}=Z_{\text {auto }}+Z_{\text {add }}$ for $i=\{a, b, c\}$. To cancel out the influence of the impedance $Z_{\text {add }}$, an impedance of opposite value $\left(-Z_{\text {add }}\right)$ is also added in series so that $Z_{m s i}=-Z_{\text {add }}$. The additional impedances $Z_{\text {add }}$ and $-Z_{a d d}$ do not affect the power flow results since they are connected in series with opposite signs, thus they cancel each other. Nevertheless, they significantly enhance the power flow convergence, enabling the compensation technique to be applied in the SVR modeling.

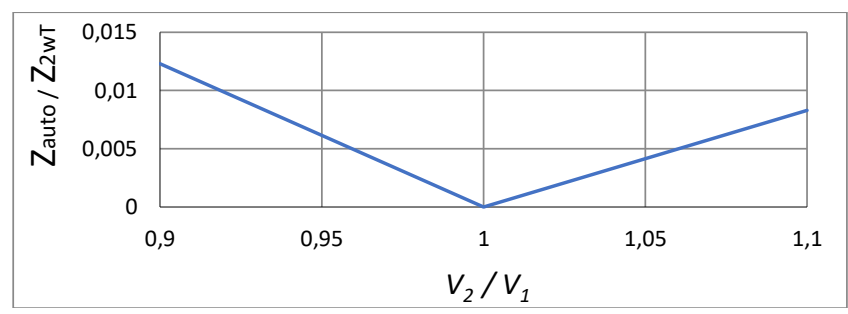

Fig. 2. Impedance ratio with respect to the output/input voltage ratio.

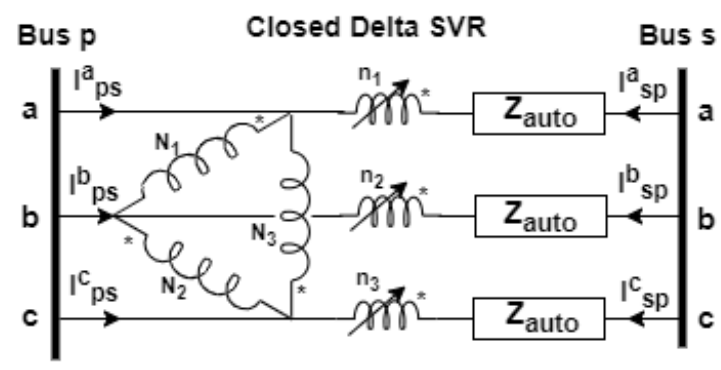

Fig. 3. Conventional 2-Bus circuit of type A SVR in closed-delta formation.

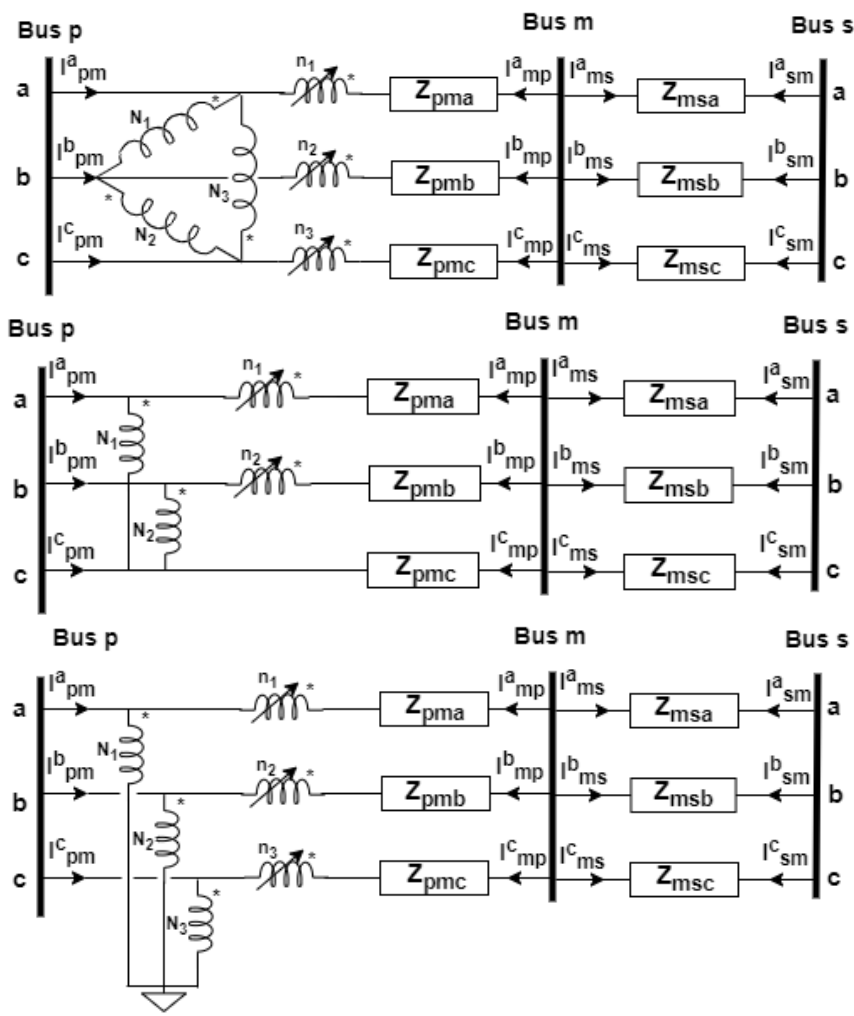

Fig. 4. Proposed 3-Bus circuits for SVRs of type A. From top to bottom: a) Closed-delta, b) Open-delta, c) Wye configuration.

By applying simple circuit analysis in Fig. 4, (5a) and (5b) are derived:

$$
\begin{aligned}
{\left[\begin{array}{c}
I_{p m} \\
I_{m p}
\end{array}\right] } & =\left[\begin{array}{cc}
\boldsymbol{Y}_{p m} \cdot\left(I_{d}+K\right) & -Y_{p m} \\
-Y_{p m} \cdot\left(I_{d}+K\right) & Y_{p m}
\end{array}\right] \cdot\left[\begin{array}{c}
V_{p} \\
V_{m}
\end{array}\right]+\left[\begin{array}{c}
I_{N} \\
0
\end{array}\right] \\
\boldsymbol{I}_{N} & =\mathbf{A} \cdot \boldsymbol{Y}_{p m} \cdot\left(-\boldsymbol{V}_{m}+\left(\boldsymbol{I}_{d}+\mathbf{K}\right) \cdot \boldsymbol{V}_{p}\right)
\end{aligned}
$$

where the matrices $\boldsymbol{A}, \boldsymbol{K}, \boldsymbol{Y}_{\boldsymbol{p m}}$ are defined in Tables 1 and 2 for all types and configurations of SVRs. $\boldsymbol{I}_{\boldsymbol{d}}$ is a $3 \times 3$ identity matrix. Eq. (5a) is used to calculate the currents $\left(\boldsymbol{I}_{\boldsymbol{p m}}, \boldsymbol{I}_{\boldsymbol{m} \boldsymbol{p}}\right)$ and voltages $\left(\boldsymbol{V}_{\boldsymbol{p}}, \boldsymbol{V}_{\boldsymbol{m}}\right)$ between the nodes $p-m$, while $(5 \mathrm{~b})$ is 
employed to calculate the current $\left(\boldsymbol{I}_{\boldsymbol{N}}\right)$ flowing through the primary windings $\left(N_{j}\right)$. Note that $(5 \mathrm{a})$ and $(5 \mathrm{~b})$ are generic and can be applied to any SVR by simply modifying the parameters $\boldsymbol{A}, \boldsymbol{K}, \boldsymbol{Y}_{\boldsymbol{p m}}$ based on the type (A or B) and configuration (closed-delta, open-delta, wye), as shown in Tables 1 and 2 . The winding ratios $\left(\frac{n_{1}}{N_{1}}, \frac{n_{2}}{N_{2}}, \frac{n_{3}}{N_{3}}\right)$ in Table 1 are related to the tap ratios $\left(\operatorname{Tap}_{j}\right)$ as follows [2]: $\frac{n_{j}}{N_{j}}=0.00625$. $\operatorname{Tap}_{j}$ for $j=\{1,2,3\}$, assuming that $\operatorname{Tap}_{j}=$ $\{-16, \ldots, 0, \ldots,+16\}$ and $-0.1<\frac{n_{j}}{N_{j}}<0.1$.

The square matrix in (5a) includes the matrix $\boldsymbol{K}$, which is not constant due to the tap variations. Therefore, it is moved outside the square matrix and (6a) is obtained, where the current sources $\boldsymbol{I}_{\boldsymbol{p}}, \boldsymbol{I}_{\boldsymbol{m}}$ are given in (6b).

$$
\begin{gathered}
{\left[\begin{array}{l}
I_{p m} \\
I_{m p}
\end{array}\right]=\left[\begin{array}{cc}
Y_{p m} & -Y_{p m} \\
-Y_{p m} & Y_{p m}
\end{array}\right] \cdot\left[\begin{array}{c}
V_{p} \\
V_{m}
\end{array}\right]+\left[\begin{array}{c}
I_{p} \\
I_{m}
\end{array}\right]} \\
{\left[\begin{array}{c}
I_{p} \\
I_{m}
\end{array}\right]=\left[\begin{array}{c}
\mathrm{A} \cdot Y_{p m} \cdot\left(-V_{m}+\left(I_{d}+\mathbf{K}\right) \cdot V_{p}\right)+Y_{p m} \cdot K \cdot V_{p} \\
-Y_{p m} \cdot K \cdot V_{p}
\end{array}\right]}
\end{gathered}
$$

Finally, the voltages and currents between the nodes $m$-s are expressed in (7), where the matrix $\boldsymbol{Y}_{\boldsymbol{m} \boldsymbol{s}}$ is given in Table 2.

$$
\left[\begin{array}{l}
I_{m s} \\
I_{s m}
\end{array}\right]=\left[\begin{array}{cc}
Y_{m s} & -Y_{m s} \\
-Y_{m s} & Y_{m s}
\end{array}\right] \cdot\left[\begin{array}{c}
V_{m} \\
V_{s}
\end{array}\right]
$$

Eqs. (6) and (7) can be comprehensively expressed in the form of an equivalent circuit as shown in Fig. 5. The parameters of the equivalent circuit for both types of SVR are given in Tables 1, 2, and 3. The square blocks of Fig. 5 consisting of $\boldsymbol{Y}_{\boldsymbol{p} \boldsymbol{m}}, \boldsymbol{Y}_{\boldsymbol{m} \boldsymbol{s}}$ are constant and are included in the $\boldsymbol{Y}_{\boldsymbol{B} U \boldsymbol{S}}$ matrix. The tap variables are comprised in the current sources, outside the $\boldsymbol{Y}_{\boldsymbol{B} U \boldsymbol{S}}$ matrix.
As shown in Table 3, the current sources depend on the matrices $\boldsymbol{Y}_{\boldsymbol{p m}}, \boldsymbol{Y}_{\boldsymbol{m} \boldsymbol{s}}$. To ensure the convergence of the power flow, the values of these matrices should be sufficiently small so that the current sources do not take an extremely large value. That is achieved by selecting a sufficiently large value of $Z_{\text {add }}$. Essentially, the role of $Z_{\text {add }}$ is to enhance the convergence by artificially increasing the value of $Z_{\text {auto }}$.

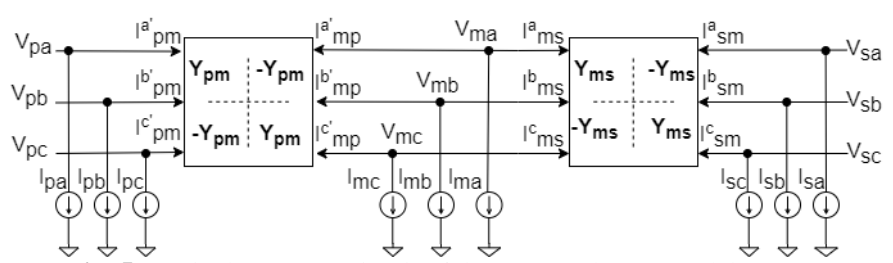

Fig. 5. Equivalent 3-Bus circuit of the proposed SVR model.

Table 1

Matrices K and A for several configurations and types of SVR

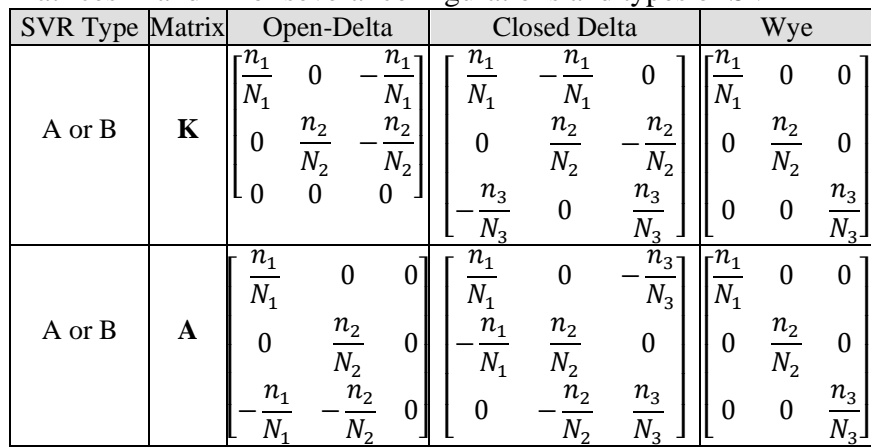

\begin{tabular}{|c|c|c|c|c|c|c|c|c|c|c|}
\hline \multicolumn{2}{|c|}{ Type Matrix } & \multicolumn{3}{|c|}{ Open-Delta } & \multicolumn{3}{|c|}{ Closed-Delta } & \multicolumn{3}{|c|}{ Wye } \\
\hline $\mathrm{A}$ & $Y_{p m}$ & $Z_{\text {auto }}+Z_{\text {add }}$ & 0 & & $Z_{\text {auto }}+Z_{\text {add }}$ & 0 & 0 & $Z_{\text {auto }}+Z_{\text {add }}$ & 0 & 0 \\
\hline B & $Y_{m s}$ & $\begin{array}{l}0 \\
0\end{array}$ & $\begin{array}{c}Z_{\text {auto }}+Z_{\text {add }} \\
0\end{array}$ & $\begin{array}{c}0 \\
Z_{\text {add }}\end{array}$ & $\begin{array}{l}0 \\
0\end{array}$ & $\begin{array}{c}Z_{\text {auto }}+Z_{\text {adc }} \\
0\end{array}$ & $\begin{array}{lc}{ }^{d} & 0 \\
Z_{\text {auto }}+Z_{\text {add }}\end{array}$ & $\begin{array}{l}0 \\
0\end{array}$ & $\begin{array}{c}Z_{\text {auto }}+Z_{\text {add }} \\
0\end{array}$ & $\begin{array}{cc}d & 0 \\
Z_{\text {quto }}+Z_{\text {add }}\end{array}$ \\
\hline $\bar{A}$ & $Y_{m s}$ & $-Z_{\text {add }}$ & 0 & $1^{-1}$ & {$[-Z$} & $d d$ & $0]^{-1}$ & {$[-Z$} & $a d d$ & $0]^{-1}$ \\
\hline B & $Y_{p m}$ & $\begin{array}{l}0 \\
0\end{array}$ & $\begin{array}{cc}-Z_{a d d} & 0 \\
0 & -Z_{a d}\end{array}$ & & $\mathrm{C}$ & $\begin{array}{c}-Z_{a d d} \\
0\end{array}$ & $\begin{array}{c}0 \\
-Z_{\text {add }}\end{array}$ & ( & $\begin{array}{c}-Z_{a d d} \\
0\end{array}$ & $\begin{array}{c}0 \\
-Z_{a d d}\end{array}$ \\
\hline
\end{tabular}

\section{Table 2}

Y matrices for several configurations and types of SVR

Table 3

Current sources for the two types of SVR

\begin{tabular}{|c|c|c|c|}
\hline SVR Type & $\boldsymbol{I}_{p}$ & $\boldsymbol{I}_{\boldsymbol{m}}$ & $\boldsymbol{I}_{s}$ \\
\hline $\mathrm{A}$ & $\mathbf{A} \cdot \boldsymbol{Y}_{p m} \cdot\left(-\boldsymbol{V}_{m}+\left(\boldsymbol{I}_{\boldsymbol{d}}+\mathbf{K}\right) \cdot \boldsymbol{V}_{\boldsymbol{p}}\right)+\boldsymbol{Y}_{p m} \cdot \boldsymbol{K} \cdot \boldsymbol{V}_{p}$ & $-\boldsymbol{Y}_{p m} \cdot \boldsymbol{K} \cdot \boldsymbol{V}_{\boldsymbol{p}}$ & $\mathbf{0}$ \\
\hline $\mathrm{B}$ & $\mathbf{0}$ & $\boldsymbol{Y}_{m s} \cdot \boldsymbol{K} \cdot \boldsymbol{V}_{s}$ & $\mathbf{A} \cdot \boldsymbol{Y}_{m s} \cdot\left(\boldsymbol{V}_{m}-\left(\boldsymbol{I}_{\boldsymbol{d}}-\mathbf{K}\right) \cdot \boldsymbol{V}_{s}\right)-\boldsymbol{Y}_{m s} \cdot \boldsymbol{K} \cdot \boldsymbol{V}_{s}$ \\
\hline
\end{tabular}

\section{Table 4}

Validation of the proposed model against Simulink for the type A SVR

\begin{tabular}{|c|c|c|c|c|c|c|c|c|c|}
\hline & \multicolumn{3}{|c|}{ Open-Delta } & \multicolumn{3}{c|}{ Closed-Delta } & \multicolumn{3}{c|}{ Wye } \\
\cline { 2 - 11 } & $|\mathbf{V 4 a b}|$ & $\mid \mathbf{V}$ 4bc $\mid$ & $\mid$ V4ca $\mid$ & $\mid$ V4ab $\mid$ & $\mid$ V4bc $\mid$ & $\mid$ V4ca $\mid$ & $\mid$ V4an $\mid$ & $\mid$ V4bn $\mid$ & $\mid$ V4cn $\mid$ \\
\hline Simulink & 13183.73427 & 12936.56756 & 13430.15426 & 13804.36230 & 13261.03079 & 13474.00390 & 7835.31967 & 7385.21215 & 7417.952216 \\
\hline Proposed & 13183.75038 & 12936.57385 & 13430.15983 & 13804.36244 & 13261.03156 & 13474.00456 & 7835.31980 & 7385.21264 & 7417.942290 \\
\hline OpenDSS & 13183.75023 & 12936.56993 & 13430.16003 & 13804.36231 & 13261.03192 & 13474.00233 & 7835.31908 & 7385.21250 & 7417.941969 \\
\hline
\end{tabular}

\begin{tabular}{|c|c|c|c|c|c|c|c|c|c|}
\hline \multirow{2}{*}{ 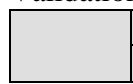 } & \multicolumn{3}{|c|}{ Open-Delta } & \multicolumn{3}{|c|}{ Closed-Delta } & \multicolumn{3}{|c|}{ Wye } \\
\hline & $\mid$ V4ab $\mid$ & V4bc & $|\mathrm{V} 4 \mathrm{ca}|$ & $\mid$ V4ab & $\mid \mathbf{V}$ 4bc & $\mid \mathbf{V}$ 4ca & |V4an & |V4bn| & $\mid \mathrm{V} 4 \mathrm{cn}$ \\
\hline
\end{tabular}

Table 5

Validation of the proposed model against Simulink for the type B SVR 


\section{Implementation of the proposed SVR model in the Z- bus power flow: A step-by-step example of a 4-Bus network}

In this section, the implementation of the proposed model is practically explained in the 4-Bus network of Fig. 6a. A closed delta SVR of type A is assumed to be connected between the nodes 2-3. $Z_{\text {auto }}$ is the SVR impedance, while $Z_{12 i}$ and $Z_{34 i}$ for $i=\{a, b, c\}$ are the line impedances connecting the buses 1-2 and 3-4, respectively.

Step 1: Modification of the network to include the proposed SVR model. The conventional SVR configuration of Fig. 6a is modified, as shown in Fig. 6b, to represent the proposed three-bus equivalent SVR model. As shown in the figure, the proposed SVR model has an additional fictitious bus 5 . Note that no load is connected to this fictitious bus. Buses 2, 5 and 3 correspond to buses $p, m$ and $s$ of the equivalent circuit of Fig. 5, respectively. The impedance $Z_{25 i}=Z_{\text {auto }}+Z_{\text {add }}$ for $i=\{a, b, c\}$, while $Z_{53 i}=-Z_{a d d}$, as explained in Section 3 . The networks of Figs. $6 \mathrm{a}$ and $6 \mathrm{~b}$ are identical since $Z_{25 i}+$ $Z_{53 i}=Z_{\text {auto }}$.

Step 2: Formulation of the $Y_{\text {BUS }}$ matrix of the network. The network of Fig. $6 \mathrm{~b}$ is described by (8), as follows:

$\left[\begin{array}{c}\boldsymbol{I}_{1} \\ \boldsymbol{I}_{2} \\ \boldsymbol{I}_{3} \\ \boldsymbol{I}_{4} \\ \boldsymbol{I}_{5}\end{array}\right]=\left[\begin{array}{ccccc}-\boldsymbol{Y}_{12} & \boldsymbol{Y}_{12} & 0 & 0 & 0 \\ \boldsymbol{Y}_{12} & -\boldsymbol{Y}_{12}-\boldsymbol{Y}_{25} & 0 & 0 & \boldsymbol{Y}_{25} \\ 0 & 0 & -\boldsymbol{Y}_{53}-\boldsymbol{Y}_{34} & \boldsymbol{Y}_{34} & \boldsymbol{Y}_{53} \\ 0 & 0 & \boldsymbol{Y}_{34} & -\boldsymbol{Y}_{34} & 0 \\ 0 & \boldsymbol{Y}_{25} & \boldsymbol{Y}_{53} & 0 & -\boldsymbol{Y}_{25}-\boldsymbol{Y}_{53}\end{array}\right] \cdot\left[\begin{array}{c}\boldsymbol{V}_{1} \\ \boldsymbol{V}_{2} \\ \boldsymbol{V}_{3} \\ \boldsymbol{V}_{4} \\ \boldsymbol{V}_{5}\end{array}\right]$

The square matrix of (8) is the $\mathrm{Y}_{\mathrm{BUS}}$ matrix of the network and it includes the $3 \times 3$ admittance matrices of the lines between the buses 1-2 $\left(\boldsymbol{Y}_{\mathbf{1 2}}\right)$ and 3-4 $\left(\boldsymbol{Y}_{\mathbf{3 4}}\right)$. Moreover, it includes the $3 \times 3$ matrices $\boldsymbol{Y}_{\mathbf{2 5}}$ and $\boldsymbol{Y}_{\mathbf{5 3}}$ calculated according to Table 2, where $\boldsymbol{Y}_{25}$ and $\boldsymbol{Y}_{\mathbf{5 3}}$ correspond to $\boldsymbol{Y}_{\boldsymbol{p m}}$ and $\boldsymbol{Y}_{\boldsymbol{m s}}$, respectively. It is noted that in (8), the voltage vectors $\boldsymbol{V}_{\boldsymbol{k}}$ for each bus $k=\{1,2,3,4,5\}$ include the three-phase voltages of bus $k$ and are mathematically expressed as $\boldsymbol{V}_{\boldsymbol{k}}=$ $\left[\begin{array}{lll}V_{k a}, & V_{k b}, & V_{k c}\end{array}\right]^{T}$.

Step 3: Formulation of the current vectors. The current vectors of (8) are given in (9).

$\boldsymbol{I}_{\mathbf{1}}=\left[\begin{array}{lll}I_{L 1 a}, & I_{L 1 b}, & I_{L 1 c}\end{array}\right]^{T}$,

$\boldsymbol{I}_{2}=\left[\begin{array}{lll}I_{L 2 a}, & I_{L 2 b}, & I_{L 2 c}\end{array}\right]^{T}+\left[\begin{array}{lll}I_{2 a}, & I_{2 b}, & I_{2 c}\end{array}\right]^{T}$,

$\boldsymbol{I}_{3}=\left[\begin{array}{lll}I_{L 3 a}, & I_{L 3 b}, & I_{L 3 c}\end{array}\right]^{T}+\left[\begin{array}{lll}I_{3 a}, & I_{3 b}, & I_{3 c}\end{array}\right]^{T}$,

$\boldsymbol{I}_{\mathbf{4}}=\left[\begin{array}{lll}I_{L 4 a}, & I_{L 4 b}, & I_{L 4 c}\end{array}\right]^{T}$

$I_{\mathbf{5}}=\left[\begin{array}{lll}I_{5 a}, & I_{5 b}, & I_{5 c}\end{array}\right]^{T}$

Each vector $\left[I_{L r a}, \quad I_{L r b}, \quad I_{L r c}\right]^{T}$ for $r=\{1,2,3,4\}$ denotes the load currents of node $r$, while $\left[\begin{array}{lll}I_{y a}, & I_{y b}, & I_{y c}\end{array}\right]^{T}$ for $y=$ $\{2,5,3\}$ denote the compensating currents calculated by Table 3 and include the tap variables of SVRs. More specifically, $\left[\begin{array}{lll}I_{2 a}, & I_{2 b}, & I_{2 c}\end{array}\right]^{T} \quad$ corresponds to $\boldsymbol{I}_{\boldsymbol{p}}, \quad\left[\begin{array}{lll}I_{5 a}, & I_{5 b}, & I_{5 c}\end{array}\right]^{T}$ corresponds to $\boldsymbol{I}_{\boldsymbol{m}}$, while $\left[\begin{array}{lll}I_{3 a}, & I_{3 b}, & I_{3 c}\end{array}\right]^{T}$ corresponds to $\boldsymbol{I}_{\boldsymbol{s}}$. Step 4: Converting the $Y_{B U S}$ matrix to a solvable form. Assuming that the bus 1 is the slack bus, the first three rows of (8) are removed and (10) is obtained, as follows:
$\left[\begin{array}{l}I_{2} \\ I_{3} \\ I_{4} \\ I_{5}\end{array}\right]=\left[\begin{array}{ccccc}Y_{12} & -Y_{12}-Y_{25} & 0 & 0 & Y_{25} \\ 0 & 0 & -Y_{53}-Y_{34} & Y_{34} & Y_{53} \\ 0 & 0 & Y_{34} & -Y_{34} & 0 \\ 0 & Y_{25} & Y_{53} & 0 & -Y_{25}-Y_{53}\end{array}\right] \cdot\left[\begin{array}{c}V_{1} \\ V_{2} \\ V_{3} \\ V_{4} \\ V_{5}\end{array}\right]$

Subsequently, the product $\left[\begin{array}{llll}\boldsymbol{Y}_{12} & \mathbf{0} & \mathbf{0} & \mathbf{0}\end{array}\right]^{T} \cdot \boldsymbol{V}_{\mathbf{1}}$ is removed from both equation sides of (10) and (11) is derived. Please note that the column $\left[\begin{array}{llll}Y_{12} & \mathbf{0} & \mathbf{0} & \mathbf{0}\end{array}\right]^{T}$ corresponds to the first column of the $\mathrm{Y}_{\mathrm{BUS}}$ matrix of (10).

$\left[\begin{array}{c}I_{2} \\ I_{3} \\ I_{4} \\ I_{5}\end{array}\right]-\left[\begin{array}{c}Y_{12} \\ 0 \\ 0 \\ 0\end{array}\right] \cdot V_{1}=\left[\begin{array}{cccc}-Y_{12}-Y_{25} & 0 & 0 & Y_{25} \\ 0 & -Y_{53}-Y_{34} & Y_{34} & Y_{53} \\ 0 & Y_{34} & -Y_{34} & 0 \\ Y_{25} & Y_{53} & 0 & -Y_{25}-Y_{53}\end{array}\right] \cdot\left[\begin{array}{c}V_{2} \\ V_{3} \\ V_{4} \\ V_{5}\end{array}\right](11)$

Step 5: Inversion of the $Y_{B U S}$ matrix. By inverting the square $\boldsymbol{Y}_{\boldsymbol{B} U \boldsymbol{S}}$ matrix at the right-hand side of (11), equation (12) is obtained.

$$
\left[\begin{array}{l}
V_{2} \\
V_{3} \\
V_{4} \\
V_{5}
\end{array}\right]^{k+1}=\left[\begin{array}{cccc}
-\boldsymbol{Y}_{12}-\boldsymbol{Y}_{25} & 0 & 0 & \boldsymbol{Y}_{25} \\
0 & -\boldsymbol{Y}_{53}-\boldsymbol{Y}_{34} & \boldsymbol{Y}_{34} & \boldsymbol{Y}_{53} \\
0 & \boldsymbol{Y}_{34} & -\boldsymbol{Y}_{34} & 0 \\
\boldsymbol{Y}_{25} & \boldsymbol{Y}_{53} & \mathbf{0} & -\boldsymbol{Y}_{25}-\boldsymbol{Y}_{53}
\end{array}\right]^{-1} \cdot\left(\left[\begin{array}{c}
\boldsymbol{I}_{2} \\
\boldsymbol{I}_{3} \\
\boldsymbol{I}_{4} \\
\boldsymbol{I}_{5}
\end{array}\right]^{k}-\left[\begin{array}{c}
\boldsymbol{Y}_{12} \\
0 \\
0 \\
0
\end{array}\right] \cdot \boldsymbol{V}_{1}\right)
$$

It is noted that the square $\boldsymbol{Y}_{\boldsymbol{B} U \boldsymbol{S}}$ matrix consists of constant elements since the tap variables of SVR are simulated as compensating currents in the current sources $\boldsymbol{I}_{2}, \boldsymbol{I}_{\mathbf{3}}, \boldsymbol{I}_{\mathbf{5}}$. Therefore, $\boldsymbol{Y}_{\boldsymbol{B} U \boldsymbol{S}}$ is inverted only once and not after each tap change. Selecting a sufficiently large value of $Z_{\text {add }}$, the current sources $\boldsymbol{I}_{2}, \boldsymbol{I}_{3}, \boldsymbol{I}_{\mathbf{5}}$ are adequately small to ensure the convergence of the power flow.

Step 6: Iterative power flow solution. Eq. (12) is iteratively solved until a certain preset tolerance is reached. In (12), $k$ denotes the iteration number. In case of a new tap variation, only the current sources $\boldsymbol{I}_{2}, \boldsymbol{I}_{3}, \boldsymbol{I}_{\mathbf{5}}$ are updated.

\section{Validation of the proposed SVR model}

\subsection{IEEE 4-Bus network with 1 SVR}

The results of the proposed model are compared against Simulink (version 2018a) and OpenDSS (version 9.1.0.1), using the IEEE 4-Bus network. Simulink and OpenDSS were selected for the validation since they are two of the most widely-used and accurate commercial simulation software tools. The former is a powerful tool performing time-domain analysis, while the latter is a well-established software for the power flow analysis of grids. In Simulink, the SVR was formed by connecting two or three ideal transformer blocks depending on the SVR configuration e.g closed-delta, opendelta, wye. The transformers have pre-specified winding ratio to correspond with those of SVR. The impedance of SVR was modeled as an external impedance connected in series with the secondary winding, as shown in Fig. 6a. A similar rationale was followed when modeling SVRs in OpenDSS.

Some clarifications about the applied parameters are provided below:

- The unbalanced loads of [17] are used in this paper. In Open-Delta and Closed-Delta configuration the loads are 
phase-to-phase connected, while in wye configuration they are phase-to-neutral.

- The SVR is placed in the position of the transformer, between the buses 2-3 [17]. The impedance of the SVR $\left(Z_{\text {auto }}\right)$ is calculated based on the data of the single-phase two-winding transformer given in [17], which has the following parameters: $V_{\text {base }}=7.2 \mathrm{kV}, S_{\text {base }}=2 \mathrm{MVA}, \mathrm{z}=$ $0.01+0.06 j(p u)$. Hence, in physical units $Z_{2 w T}=0.26+$ $1.55 \mathrm{j} \mathrm{Ohm}$. According to the analysis of section $2, Z_{\text {auto }} \leq$ $0.01 \cdot Z_{2 w T}$, thus $Z_{\text {auto }} \leq 0.0026+0.0155 \mathrm{j} \mathrm{Ohm}$. It is noted, that although the SVR impedance varies with the tap settings of SVR, we assume it is constant. The inaccuracy introduced by this assumption is negligible, due to the very low value of $Z_{\text {auto }}$.

- The taps applied in the simulation are as follows: $\left(\operatorname{Tap}_{1}\right.$, $\left.\operatorname{Tap}_{2}, \operatorname{Tap}_{3}\right)=(16,8,8)$. Note that in open delta configuration $\mathrm{Tap}_{3}$ does not exist.

The results of the proposed model, Simulink and OpenDSS are contrasted in Table 4 for the SVR of type A, and in Table 5 for the SVR of type B. Indicatively, only the phase-to-phase (or phase-to-neutral) voltages of the $4^{\text {th }}$ bus are depicted here. The remaining buses present similar accuracy. As shown, the results of the proposed approach are in full agreement with those of Simulink and OpenDSS confirming the accuracy of the proposed model.

\subsection{8-Bus network with 3 SVRs}

The proposed SVR model is further validated in the 8-Bus network of Fig. 7, consisting of 3 SVRs and 4 unbalanced constant impedance loads. It is a 4-wire network with the following assumptions: 1) every bus is considered perfectly grounded, 2) the mutual impedance between the neutral and phase conductors is zero. The parameters of the network are quoted in Table 6, while the loads are given in Table 7. The taps of SVRs for the simulated configurations are presented in Table 8.
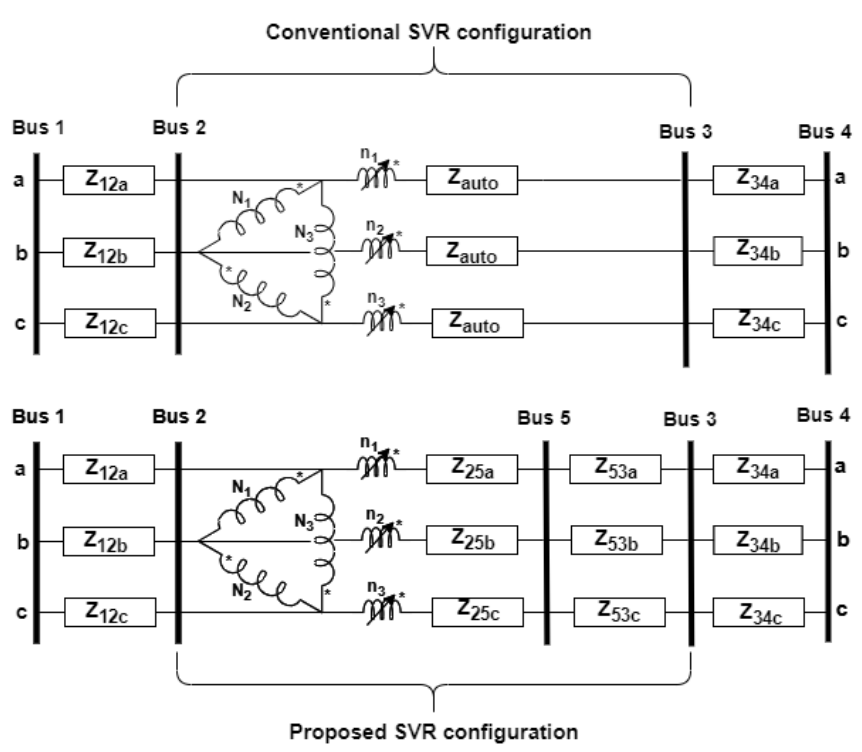

Fig. 6. From top to bottom: a) 4-Bus network with the conventional SVR configuration between the buses 2-3 and b) 4-Bus network with the proposed SVR modeling.

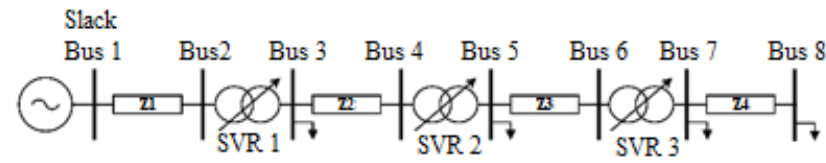

Fig 7. Unbalanced 8-Bus MV network consisting of 4 constant impedance loads and 3 SVRs.

The proposed approach is compared against Simulink and OpenDSS and the results are presented in Table 9. Indicatively, only the voltage magnitude of the three phases of bus 8 are depicted, nevertheless, the remaining buses present similar accuracy. In Table 9, the results of the proposed approach are presented in regular font, the results of Simulink in bold, while the results of OpenDSS in green color. As shown, although the network consists of 3 SVRs, the proposed model produces almost identical steady-state power flow results with Simulink and OpenDSS confirming its accuracy. Finally, the currents of the lines of the 8-bus network are shown in Table 10 for the proposed approach (regular font), Simulink (bold) and OpenDSS (green). The SVRs are in closed-delta connection of type A and their tap settings are provided in Table 8 . It is confirmed that the proposed approach is very accurate since all results are identical with those of commercial software tools.

Table 6

Parameters of 8-Bus network

\begin{tabular}{|c|c|}
\hline Distance of the lines & $5 \mathrm{~km}$ \\
\hline Phase-to-neutral Voltage of slack bus & $7200 \mathrm{~V}$ \\
\hline Frequency of the network & $50 \mathrm{~Hz}$ \\
\hline Resistance of the lines (per phase) & $0.4 \Omega / \mathrm{km}$ \\
\hline Self-reactance of the lines (per phase) & $0.3 \Omega / \mathrm{km}$ \\
\hline Mutual-reactance between phase conductors & $0.1 \Omega / \mathrm{km}$ \\
\hline Impedance of SVRs & $0 \mathrm{Ohm}$ \\
\hline
\end{tabular}

Table 7

Loads of 8-Bus network

\begin{tabular}{|c|c|}
\hline \multirow{2}{*}{ Load Bus 3 } & $Z_{L a}=250 \Omega$ in parallel with $j 1000 \Omega$ \\
& $Z_{L b}=250 \Omega$ in parallel with $j 1000 \Omega$ \\
& $Z_{L c}=250 \Omega$ in parallel with $j 1000 \Omega$ \\
\hline \multirow{2}{*}{ Load Bus 5 } & $Z_{L a}=250 \Omega$ in parallel with $j 1000 \Omega$ \\
& $Z_{L b}=125 \Omega$ in parallel with $j 500 \Omega$ \\
& $Z_{L c}=250 \Omega$ in parallel with $j 1000 \Omega$ \\
\hline \multirow{2}{*}{ Load Bus 7 } & $Z_{L a}=150 \Omega$ in parallel with $j 600 \Omega$ \\
& $Z_{L b}=100 \Omega$ in parallel with $j 400 \Omega$ \\
& $Z_{L c}=150 \Omega$ in parallel with $j 600 \Omega$ \\
\hline \multirow{2}{*}{ Load Bus 8 } & $Z_{L a}=250 \Omega$ in parallel with $j 1000 \Omega$ \\
& $Z_{L b}=250 \Omega$ in parallel with $j 1000 \Omega$ \\
& $Z_{L c}=250 \Omega$ in parallel with $j 1000 \Omega$ \\
\hline
\end{tabular}

Table 8

Taps of SVRs $\left(\operatorname{Tap}_{1}, \mathrm{Tap}_{2}, \mathrm{Tap}_{3}\right)$ for the simulated configurations

\begin{tabular}{|c|c|c|c|}
\hline WVe Type A & $(8,10,8)$ & $(8,10,8)$ & $(8,10,8)$ \\
\hline Open Delta Type A & $(8,8,-)$ & $(8,8,-)$ & $(8,8,-)$ \\
\hline Close Delta Type A & $(8,10,8)$ & $(8,10,8)$ & $(8,10,8)$ \\
\hline Wye Type B & $(8,10,8)$ & $(8,10,8)$ & $(8,10,8)$ \\
\hline Open Delta Type B & $(8,8,-)$ & $(8,8,-)$ & $(8,8,-)$ \\
\hline Close Delta Type B & $(8,10,8)$ & $(8,10,8)$ & $(8,10,8)$ \\
\hline
\end{tabular}

Table 9

Phase-to-neutral voltages for the 8-Bus network calculated with the proposed method, Simulink and OpenDSS for several SVR configurations

\begin{tabular}{|c|c|c|c|}
\hline & Phase A & Phase B & Phase C \\
\hline Wye & 7331.1433 & 7244.2791 & 7450.4267 \\
\hline
\end{tabular}




\begin{tabular}{|c|c|c|c|}
\hline Type A & $\mathbf{7 3 3 1 . 1 8 3 1}$ & $\mathbf{7 2 4 4 . 3 4 6 2}$ & $\mathbf{7 4 5 0 . 4 7 1 2}$ \\
& 7331.0209 & $\mathbf{7 2 4 4 . 2 3 0 3}$ & $\mathbf{7 4 5 0 . 3 0 2 9}$ \\
\hline Open Delta & 7784.6505 & 7576.6246 & 6468.4454 \\
Type A & $\mathbf{7 7 8 4 . 6 9 3 6}$ & $\mathbf{7 5 7 6 . 7 0 1 3}$ & $\mathbf{6 4 6 8 . 4 7 2 9}$ \\
& 7784.6967 & 7576.5696 & $\mathbf{6 4 6 8 . 5 0 9 1}$ \\
\hline Close Delta & 7799.5715 & 7845.3272 & 7880.3313 \\
Type A & $\mathbf{7 7 9 9 . 5 7 0 4}$ & $\mathbf{7 8 4 5 . 3 5 2 1}$ & $\mathbf{7 8 8 0 . 3 7 8 2}$ \\
& 7799.4528 & 7845.2551 & $\mathbf{7 8 8 0 . 1 9 0 0}$ \\
\hline Wye & 7377.7522 & 7312.6066 & 7500.9205 \\
Type B & $\mathbf{7 3 7 7 . 7 2 0 9}$ & $\mathbf{7 3 1 2 . 6 1 4 4}$ & $\mathbf{7 5 0 0 . 9 6 5 6}$ \\
& 7377.6269 & 7312.5579 & $\mathbf{7 5 0 0 . 7 9 5 6}$ \\
\hline Open Delta & 7858.3033 & 7649.9001 & 6463.4446 \\
Type B & $\mathbf{7 8 5 8 . 3 4 7 0}$ & $\mathbf{7 6 4 9 . 9 7 9 6}$ & $\mathbf{6 4 6 3 . 4 7 1 7}$ \\
\hline Close Delta & 7858.1504 & 7649.7734 & $\mathbf{6 4 6 3 . 4 8 8 5}$ \\
Type B & $\mathbf{7 8 7 4 . 3 1 4 1}$ & $\mathbf{7 9 6 0 . 3 6 9 5}$ & 7948.5619 \\
& 7874.3046 & $\mathbf{7 9 6 0 . 3 9 4 6}$ & $\mathbf{7 9 4 8 . 6 0 6 9}$ \\
\hline
\end{tabular}

Table 10

Line currents for the 8-Bus network calculated with the proposed method, Simulink and OpenDSS for the closed-delta configuration

\begin{tabular}{|c|c|c|c|}
\hline & Phase A & Phase B & Phase C \\
\hline \multirow{3}{*}{ Line 1-2 } & 175.6681 & 248.2772 & 186.0943 \\
& $\mathbf{1 7 5 . 6 6 4 3}$ & $\mathbf{2 4 8 . 2 7 2 4}$ & $\mathbf{1 8 6 . 0 9 0 1}$ \\
& $\mathbf{1 7 5 . 6 8 0 0}$ & $\mathbf{2 4 8 . 2 8 3 0}$ & $\mathbf{1 8 6 . 1 1 3 0}$ \\
\hline & 133.2405 & 199.8065 & 139.1992 \\
Line 3-4 & $\mathbf{1 3 3 . 2 3 7 7}$ & $\mathbf{1 9 9 . 8 0 2 6}$ & $\mathbf{1 3 9 . 1 9 6 0}$ \\
& $\mathbf{1 3 3 . 2 4 8 0}$ & $\mathbf{1 9 9 . 8 1 0 0}$ & $\mathbf{1 3 9 . 2 1 0 0}$ \\
\hline & 92.8176 & 123.2700 & 95.2407 \\
Line 5-6 & $\mathbf{9 2 . 8 1 5 6}$ & $\mathbf{1 2 3 . 2 6 7 7}$ & $\mathbf{9 5 . 2 3 8 5}$ \\
& $\mathbf{9 2 . 8 2 0 7}$ & $\mathbf{1 2 3 . 2 7 2 0}$ & $\mathbf{9 5 . 2 4 4 9}$ \\
\hline \multirow{3}{*}{ Line 7-8 } & 32.1585 & 32.3471 & 32.4914 \\
& $\mathbf{3 2 . 1 5 7 7}$ & $\mathbf{3 2 . 3 4 6 4}$ & $\mathbf{3 2 . 4 9 0 6}$ \\
& $\mathbf{3 2 . 1 5 8 0}$ & $\mathbf{3 2 . 3 4 6 8}$ & 32.4909 \\
\hline
\end{tabular}
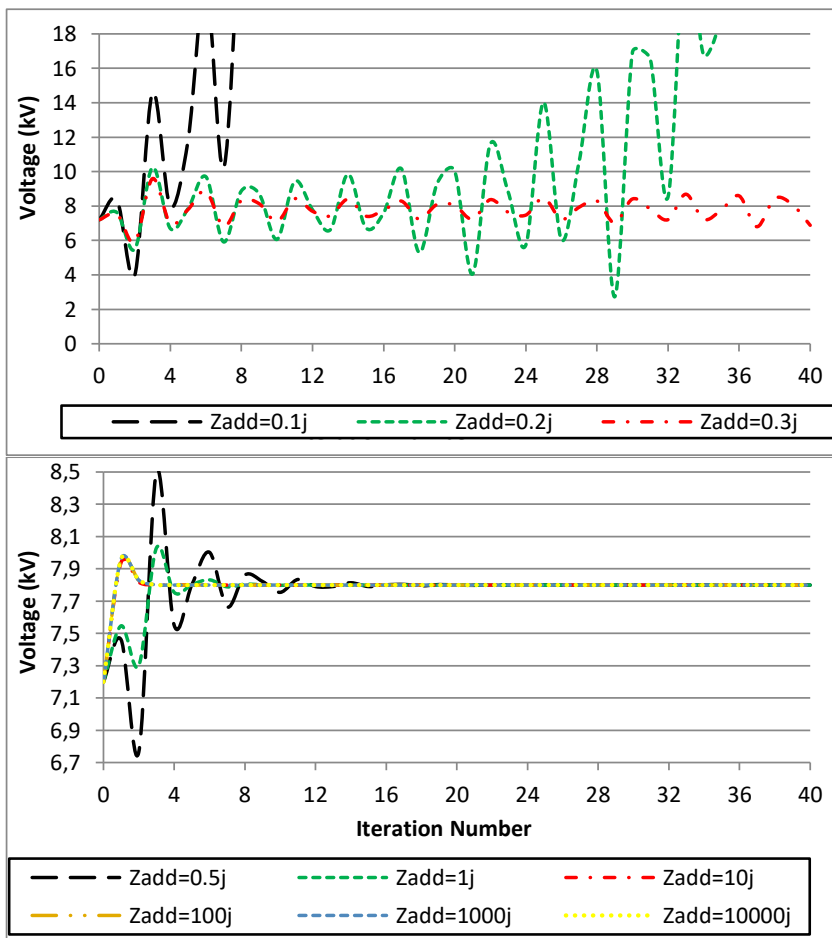

Fig. 8. Voltage of phase A of the last bus of the 8-bus network, during the iterative power flow process. SVRs are in closed-delta and type A connection. From top to bottom: a) low Zadd values, b) large Zadd values

\subsection{Convergence properties of the proposed SVR model}

The convergence characteristics of the proposed model are presented here, with respect to the values of the additional impedance $Z_{\text {add }}$ introduced in Section 3. Fig 8 depicts indicatively the voltage of phase A at the last bus of the 8-bus network, throughout the iterative process of power flow, for several values of $Z_{\text {add }}$. More specifically, Fig. 8a depicts the voltage for low values of $Z_{\text {add }}$ (from $0.1 \mathrm{j}$ to $0.3 \mathrm{j}$ ), while Fig. $8 \mathrm{~b}$ for high values (from $0.5 \mathrm{j}$ to $10000 \mathrm{j}$ ). Although $\mathrm{Z}_{\mathrm{add}}$ takes only inductive values in Fig. 8, similar results are derived with resistive values of similar magnitude. It is clarified that a flat start was considered for all the nodes of the network. Based on the figure, the following observations are derived:

- For low $Z_{\text {add }}$ values (see Fig. 8a) the Z-Bus power flow diverges. It occurs because for low $Z_{\text {add }}$ values, the matrices $\boldsymbol{Y}_{\boldsymbol{p} \boldsymbol{m}}, \boldsymbol{Y}_{\boldsymbol{m} \boldsymbol{s}}$ are increased (see Table 2). As a result, the current sources of Fig. 5 (see Table 3) are increased as well, resulting in the divergence of the power flow.

- The algorithm presents fast convergence for all $Z_{\text {add }} \geq 0.5 j$, as shown in Fig. 8b. Thus, $Z_{\text {add }}$ can be arbitrarily selected over a very wide range of values, making the selection of a proper $Z_{\text {add }}$ value a very simple process.

- The algorithm converges to the same solution for all $Z_{\text {add }} \geq$ $0.5 j$. This is logical since an impedance $-Z_{\text {add }}$ is connected in series with $Z_{\text {add }}$ (see section 3 ), canceling completely the influence of $Z_{\text {add }}$. In fact, $Z_{\text {add }}$ is added to reduce the values of the current sources of Table 3, and therefore, enhance the convergence of the power flow, without affecting the power flow results.

\section{Performance of the proposed model in a real network with various local controllers}

\subsection{Description of the network}

The performance of the proposed SVR model is tested in the IEEE 8500-Node network, which is a real large-scale distribution network. It includes originally 4 SVRs and 4 capacitor banks. SVR 1 is assumed to be connected in wye configuration, SVR 2 in open delta configuration, while SVRs 3 and 4 are connected in closed delta configuration. SVR 1, SVR 2, SVR 3 and SVR 4 are connected between the buses 1-2, 405-406, 1057-1058 and 1311-1312, respectively. The capacitors have been connected in buses 15, 146, 644 and 1298. The topology of IEEE 8500-network is shown in Fig. 9. In order to investigate the interactions of SVRs with the DGs, we connected 4 DGs to the buses 100, 350, 835 and 1600 .

To get a sense about the position of each bus inside the network, their distance from the substation is presented in Fig. 10. More details about the data of the network e.g lines, loads are provided in [17]. All buses of the network are considered perfectly grounded.

\subsection{Modeling of the local voltage controllers}

Switched capacitors in distribution networks usually operate in voltage or reactive power control. In voltage control mode, the controller switches the capacitor ON when the measured voltage is less than a minimum value and OFF when it is more than a maximum value [24]. In reactive power mode, the capacitor is switched depending on the reactive power value and direction through the supervised line. In 
power flow formulation, capacitors are simply modeled as constant impedance loads with $Z_{c}=(j \omega C)^{-1}$. In this study, all capacitor banks are assumed to operate in voltage control mode, and all have the same capacitances. The capacitance of each phase is given in Table 12 .

The steady state modeling of DGs is usually categorized depending on the generated voltage/current profile as well as the power profile, as follows:

1. Voltage and current profile. Inverter-based DGs can generate balanced phase-to-phase voltage, balanced phase-to-neutral voltage, or balanced current. On the contrary, the synchronous generator-based DGs present nonzero finite negative- and zero-sequence admittances, thus they usually generate unbalanced voltages and currents [6].

2. Power profile. DGs can operate in constant $\mathrm{PQ}$, constant voltage (conventional PV bus modeling), or constant P$\mathrm{Q}(\mathrm{V})$ mode [25] [26].

In this study, the DGs are assumed to generate different current and voltage profiles as shown in Table 11. The active power and the droop gains of DGs 2 and 3 are provided in Table 12.

\subsection{Switching delays of local voltage controllers}

LVCs are usually configured to react with pre-specified time delays. This is done in order to coordinate the switching actions of LVCs and to avoid unnecessary switchings, due to temporary voltage variations.

The switching capacitors are set with an intentional time delay such that the capacitor switching is activated, after a pre-specified time delay, from the instant that a voltage violation is sensed [18] [19] [27]. The intentional time delay, as well as the reference voltage and bandwidth of capacitors are quoted in Table 12.

Similarly, SVRs step the voltage up or down when a voltage violation occurs beyond a pre-specified bandwidth, considering a pre-specified time delay. More specifically, as soon as a voltage violation is detected, the intentional time delay is counted before the first step-up/down switching action. If the voltage remains outside the bandwidth, additional step-up/down actions are executed with a mechanical delay [18] [19]. The intentional and mechanical time delay, as well as the reference voltage and bandwidth of SVRs are quoted in Table 12.

DGs present a very fast reaction time compared to the intentional or mechanical delays of the other LVCs. Thus, it is assumed that they react instantaneously to any load/generation variation [18] [19] [27]-[30].

It is clarified that the intentional time delays of capacitors and SVRs were selected based on their distance from substation. Near to substation LVCs have shorter intentional time delays [31, page 29], while delays are increased for longer distances.

Switching capacitors, SVRs, and OLTCs can operate in two different ways: a) their three-phases are independently controlled; thus, each device has three local controllers acting independently on each phase, or b) each device has one local controller, which controls the three phases simultaneously
[32]-[34]. In this study, we assume that each phase is independently controlled.

\subsection{Power flow algorithms for distribution networks with local controllers}

The inclusion of the LVCs into the power flow is known to create multiple power flow solutions, as a result of the different reaction speed of LVCs and the discrete switching states of SVRs, OLTCs and capacitor banks. The state of-theart for simulating local voltage controllers computes the most likely operational solution by considering the different precedence of the controlled devices, according to their reaction time delays [11].

The time delays of LVCs were introduced for the first time into the power flow studies in 2000 by a research group of Siemens [35] and it is considered the state-of-the-art so far in the steady state modelling of distribution networks with LVCs. More specifically, LVCs were divided into different groups based on their switching time delays. The LVCs with the fastest reaction belong to the first group, while the slowest ones belong to the last group. The method of [35] was improved later in [7] and [11] by introducing sensitivity parameters to accelerate the convergence of the power flow. In the aforementioned approaches, a single-phase power flow is applied neglecting the network unbalances.

Authors in [18] [19] developed a three-phase power flow algorithm, which precisely considers the actual switching sequence of LVCs based on their switching time delays. Moreover, it considers the various operational modes of DGs as well as the unbalances that usually exist in distribution networks. In this case study, we applied the method of [18] [19] for calculating the power flow of the examined 8500node network considering the LVCs and DG characteristics of Tables 11 and 12.

\subsection{Power flow results}

Initially, the power flow is executed considering all the LVCs at their initial switching state. In this example, the taps of all SVRs are initially assumed at 0 position, while the capacitors are OFF. After the convergence of the power flow (at $43^{\text {th }}$ iteration), the voltages of LVCs are checked and a switching action is undertaken from the LVCs with the shortest time delay, whose voltage bandwidth was violated. Then, the power flow is executed again, the voltages of LVCs are re-checked and a switching action is activated in order of priority, until all voltages lie inside their bandwidth and no other switching actions are required.

All switching actions of the examined network are summarized in Table 13 and explained below. Furthermore, the phase voltages of SVR 1, SVR 3 and capacitor 4 as well as the reactive power of DGs during the power flow process are indicatively depicted in Figs 11-14. The voltages of the other LVCs are not depicted due to space limitation. The algorithm converges in 309 iterations. The proposed model as well as the three-phase model of [1] are applied for the modelling of SVRs and the results of both models are discussed in the next sub-section.

Switching action 1: The first switching action is taken over by the SVR 1 since it violates its voltage bandwidth and has 
the shortest time delay. More specifically, as shown in Fig. 11 , after the power flow has converged at $43^{\text {th }}$ iteration, the voltage of each phase of SVR 1 is near $7200 \mathrm{~V}$, which is below the bandwidth of $7465 \mathrm{~V}-7535 \mathrm{~V}$. Therefore, SVR 1 step up the voltage of all phases by one tap in order to increase its voltage toward its bandwidth.

Switching actions 2-6: After the first switching action of SVR 1 , its voltage remains below the bandwidth. Therefore, SVR1 steps up the voltage of all phases sequentially, with a mechanical time delay, until the voltages of all phases lie inside bandwidth, as shown in Fig. 11.

Switching actions 7: After the switching actions of SVR 1, the voltage of SVR 2 lies inside the bandwidth and no switching is performed by SVR 2. On the opposite, the phase voltages of SVR 3 remain outside the bandwidth resulting in a step-up function by SVR 3, after the time delay of $60 \mathrm{sec}$ (the time delay of SVR 3).

Switching actions 8-10: The voltage of SVR 3 still remains below its bandwidth and additional step-up functions are performed with a mechanical time delay for the phases that violate the voltage, as depicted in Fig. 12.

Switching actions 11: The three phases of capacitor bank 4 are switched ON after the time delay of 75 seconds (it corresponds to $213^{\text {th }}$ iteration), as shown in Fig. 13.

Switching actions 12: The three-phases of SVR 4 lie outside the bandwidth resulting in a switching action after the time delay of 90 seconds.

Switching actions 13-16: SVR 4 undertakes sequential switching actions with a mechanical time delay for the phases with a violated voltage, until all voltages are inside bandwidth.

Switching actions 17: At 135s, phase B of capacitor 2 is switched ON. It occurs because the step-up function of SVR 4 at 90 s caused a reduction at the reactive power of DG 4 (see Fig. 14), which in turn forced the voltage of phase B of capacitor 2 below its bandwidth. As a result, a switching action is taken over from Capacitor 2 after the time delay of $45 \mathrm{~s}$, namely at $135 \mathrm{~s}$.

Switching actions 18: Similarly, with the switching action 17, the switching action 18 is activated at $139 \mathrm{~s}$, due to the voltage violation of phase A of capacitor 2 at $94 \mathrm{~s}$.

Switching actions 19: The step-up action of SVR 4 at 92s causes a reduction of the reactive power of DG 4 (see Fig. 14), which in turn causes a voltage violation of phases A and B of SVR 3, as shown in Fig. 12. As a result, a step-up function is performed by the phases $\mathrm{A}$ and $\mathrm{B}$ after the time delay of $60 \mathrm{~s}$, namely at $152 \mathrm{~s}$.

Switching actions 20-22: SVR 3 undertakes additional stepup functions with a mechanical delay until the voltage of all phases lie inside the bandwidth, as depicted in Fig. 12.

Switching actions 23: Due to the switching action of phase B of capacitor 2 at $135 \mathrm{~s}$, the reactive power of DG 1 (see Fig. 14 ) is reduced forcing the voltage of phase $C$ of capacitor 2 below its bandwidth. Thus, phase $\mathrm{C}$ of capacitor 2 is switched ON 45s later at 180 s.

\subsection{Discussion about the power flow results}

The voltage profile of the network after all LVC switching actions have been executed is depicted in Fig. 15, using the proposed model as well as the model of ref. [1] for the simulation of SVRs. The two models produce exactly the same results confirming the accuracy of the proposed model.

However, a huge difference is observed in the computation time of the two models. As shown in Table 13, 19 switching actions are executed in the examined case study by the SVRs until their voltage is stabilized inside their bandwidths. The model of [1] includes the taps of SVRs in the $\mathrm{Y}_{\mathrm{BUS}}$ matrix, and therefore, the re-factorization (or inversion) of the $\mathrm{Y}_{\mathrm{BUS}}$ matrix is executed 19 times during the power flow process On the contrary, the proposed model simulates the taps as fictitious current sources and the $\mathrm{Y}_{\mathrm{BUS}}$ matrix remains constant throughout the whole power flow process. As a result, the power flow is executed in only $154.5 \mathrm{~s}$ using the proposed SVR model ( $0.5 \mathrm{~s}$ per iteration for 309 iterations) and in $1408.5 \mathrm{~s}$ using the model of [1]. It is clarified that the inversion of the $\mathrm{Y}_{\mathrm{BUS}}$ matrix of the examined network is performed in around 66s in Matlab, due to the large size of the network. A summary of the computation time of the power flow for the two SVR models is provided in Table 14. All simulations were conducted in a PC with an Intel Core i7, 3.4GHz CPU and 16GB DDR3 RAM.

Finally, another important thing to note in Figs 11-14 is the fast convergence of the power flow when the proposed SVR model is applied. More specifically, the power flow converges very fast to the new solution after each SVR tap variation. Although the proposed model uses fictitious current sources to simulate the taps of SVRs, the convergence of power flow is not sacrificed.

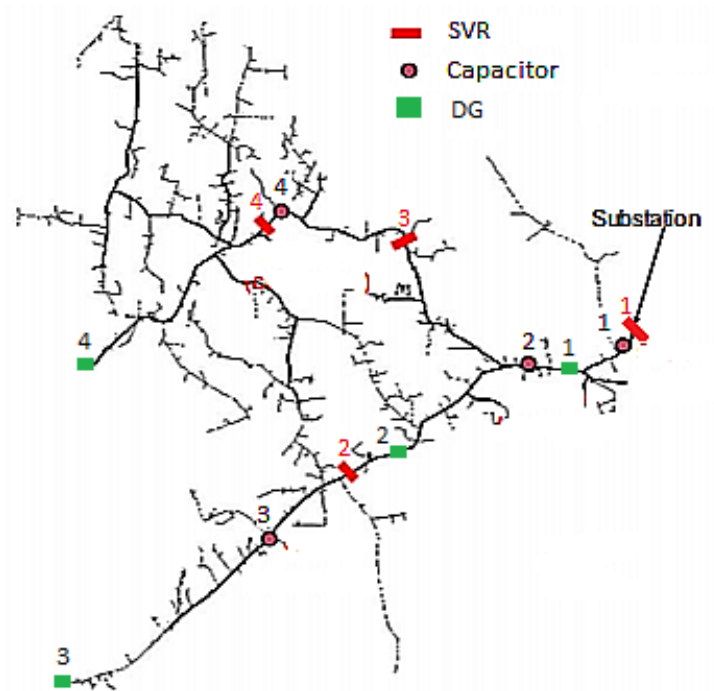

Fig 9. The IEEE 8500-node network. It consists of 4 capacitor banks and 4 SVRs. The outputs of the SVRs are connected to the following buses: SVR $1 \rightarrow$ Bus 2, SVR $2 \rightarrow$ Bus 406, SVR $3 \rightarrow$ Bus 1058, SVR $4 \rightarrow$ Bus 1312 . The capacitors are connected as follows: CAP $1 \rightarrow$ Bus 15, CAP $2 \rightarrow$ Bus 146, CAP $3 \rightarrow$ Bus 644, CAP $4 \rightarrow$ Bus 1298. The DGs are connected as follows: DG $1 \rightarrow$ Bus 100, DG $2 \rightarrow$ Bus 350, DG $3 \rightarrow$ Bus 835, DG $4 \rightarrow$ Bus 1600 .

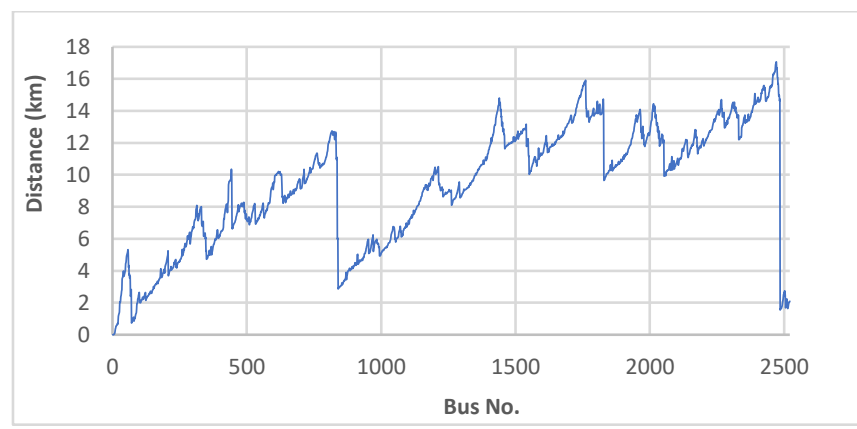


Fig 10. Distance of the buses of the IEEE 8500-node network from the substation [6, supplementary material].

Table 11

DG characteristics

DG \# Power profile Connecting Bus $\quad$ Voltage/Current profile

\begin{tabular}{|c|c|c|c|}
\hline 1 & Constant PV & 100 & SG (Unbalanced voltage and current) \\
\hline 2 & Droop Q(V) & 350 & Balanced Current \\
\hline 3 & Droop Q(V) & 835 & Balanced Current \\
\hline 4 & Constant PV & 1600 & Balanced Voltage \\
\hline
\end{tabular}

Table 12

Parameters of the modified IEEE 8500-Node Network

\begin{tabular}{|c|c|}
\hline Data about the lines & Given in [13] \\
\hline Data about the loads & Given in [13] \\
\hline Voltage of slack bus & $7200 \mathrm{~V}$ \\
\hline Frequency of the network & $50 \mathrm{~Hz}$ \\
\hline Reference voltage of SVRs & $7500 \mathrm{~V}$ \\
\hline Bandwidth of SVRs & $70 \mathrm{~V}$ \\
\hline Intentional delay of SVR 1 & $15 \mathrm{~s}$ \\
\hline Intentional delay of SVR 2 & $60 \mathrm{~s}$ \\
\hline Intentional delay of SVR 3 & $60 \mathrm{~s}$ \\
\hline Intentional delay of SVR 4 & $90 \mathrm{~s}$ \\
\hline Mechanical delay of SVRs & $2 \mathrm{~s}$ \\
\hline Intentional delay of CAP 1 & $30 \mathrm{~s}$ \\
\hline Intentional delay of CAP 2 & $45 \mathrm{~s}$ \\
\hline Intentional delay of CAP 3 & $75 \mathrm{~s}$ \\
\hline Intentional delay of CAP 4 & $75 \mathrm{~s}$ \\
\hline Reference voltage of CAPs & $7600 \mathrm{~V}$ \\
\hline Bandwidth of CAPs & $300 \mathrm{~V}$ \\
\hline Capacitance of each phase & $2 \cdot 10^{-5} \mathrm{~F}$ \\
\hline Active power of DGs & $2 \mathrm{MW}$ \\
\hline Apparent power limit of DG $\mathbf{1}$ & $13 \mathrm{MVA}$ \\
\hline Apparent power limit of DG $\mathbf{~ \& ~} \mathbf{3}$ & $3 \mathrm{MVA}$ \\
\hline Apparent power limit of DG 4 & $10 \mathrm{MVA}$ \\
\hline Reference voltage of DGs $\left(\boldsymbol{V}_{\boldsymbol{r e f}}\right)$ & $7500 \mathrm{~V}$ \\
\hline Droop gain of DGs $\mathbf{2}$ \& 3 $\left.\left(\boldsymbol{K}_{\boldsymbol{q}}\right) \mathbf{2 5}\right]$ & $10^{-4} \mathrm{~V} / \mathrm{Var}$ \\
\hline
\end{tabular}

Table 13

Switching actions of the local voltage controllers of IEEE 8500node network.

\begin{tabular}{|c|c|c|c|c|c|}
\hline $\begin{array}{c}\text { Number of } \\
\text { switching action }\end{array}$ & \begin{tabular}{|c|} 
Local \\
Controller
\end{tabular} & Phase & $\begin{array}{c}\text { Switching } \\
\text { time }\end{array}$ & $\begin{array}{c}\text { Type of } \\
\text { switching }\end{array}$ & $\begin{array}{c}\text { Iteration } \\
\text { No. }\end{array}$ \\
\hline 1 & SVR 1 & $\mathrm{ABC}$ & $15 \mathrm{~s}$ & Step Up & 43 \\
\hline 2 & SVR 1 & $\mathrm{ABC}$ & $17 \mathrm{~s}$ & Step Up & 68 \\
\hline 3 & SVR 1 & $\mathrm{ABC}$ & $19 \mathrm{~s}$ & Step Up & 91 \\
\hline 4 & SVR 1 & $\mathrm{ABC}$ & $21 \mathrm{~s}$ & Step Up & 111 \\
\hline 5 & SVR 1 & $\mathrm{ABC}$ & $23 \mathrm{~s}$ & Step Up & 131 \\
\hline 6 & SVR 1 & $\mathrm{ABC}$ & $25 \mathrm{~s}$ & Step Up & 148 \\
\hline 7 & SVR 3 & $\mathrm{ABC}$ & $60 \mathrm{~s}$ & Step Up & 165 \\
\hline 8 & SVR 3 & $\mathrm{ABC}$ & $62 \mathrm{~s}$ & Step Up & 180 \\
\hline 9 & SVR 3 & $\mathrm{ABC}$ & $64 \mathrm{~s}$ & Step Up & 193 \\
\hline 10 & SVR 3 & $\mathrm{AB}$ & $66 \mathrm{~s}$ & Step Up & 204 \\
\hline 11 & CAP 4 & $\mathrm{ABC}$ & $75 \mathrm{~s}$ & Switch ON & 213 \\
\hline 12 & SVR 4 & $\mathrm{ABC}$ & $90 \mathrm{~s}$ & Step Up & 222 \\
\hline 13 & SVR 4 & $\mathrm{AB}$ & $92 \mathrm{~s}$ & Step Up & 229 \\
\hline 14 & SVR 4 & $\mathrm{AB}$ & $94 \mathrm{~s}$ & Step Up & 236 \\
\hline 15 & SVR 4 & $\mathrm{~A}$ & $96 \mathrm{~s}$ & Step Up & 242 \\
\hline 16 & SVR 4 & $\mathrm{~A}$ & $98 \mathrm{~s}$ & Step Up & 248 \\
\hline 17 & CAP 2 & $\mathrm{~B}$ & $135 \mathrm{~s}$ & Switch ON & 254 \\
\hline 18 & CAP 2 & $\mathrm{~A}$ & $139 \mathrm{~s}$ & Switch ON & 261 \\
\hline 19 & SVR 3 & $\mathrm{AB}$ & $152 \mathrm{~s}$ & Step Up & 269 \\
\hline 20 & SVR 3 & $\mathrm{~A}$ & $154 \mathrm{~s}$ & Step Up & 275 \\
\hline 21 & SVR 3 & $\mathrm{~A}$ & $156 \mathrm{~s}$ & Step Up & 271 \\
\hline 22 & SVR 3 & $\mathrm{~A}$ & $158 \mathrm{~s}$ & Step Up & 287 \\
\hline 23 & CAP 2 & $\mathrm{C}$ & $180 \mathrm{~s}$ & Switch ON & 293 \\
\hline
\end{tabular}

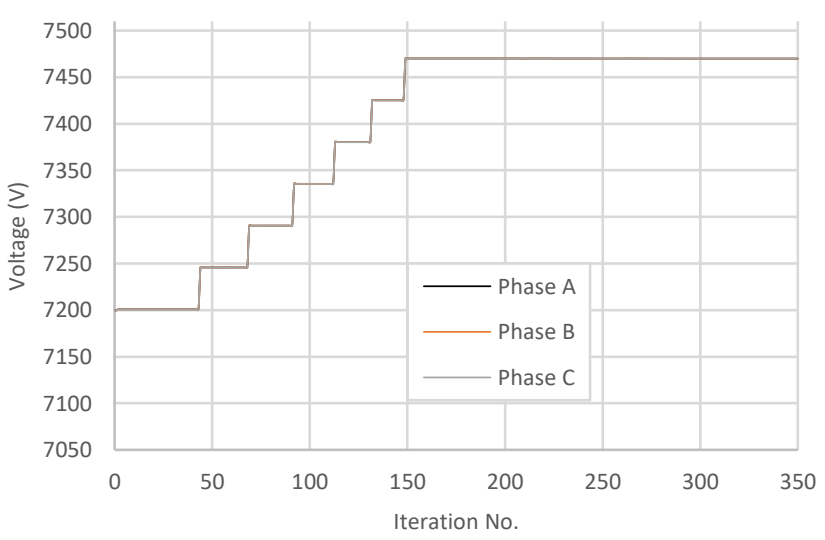

Fig 11. Voltage of SVR 1 throughout the iterative power flow process.

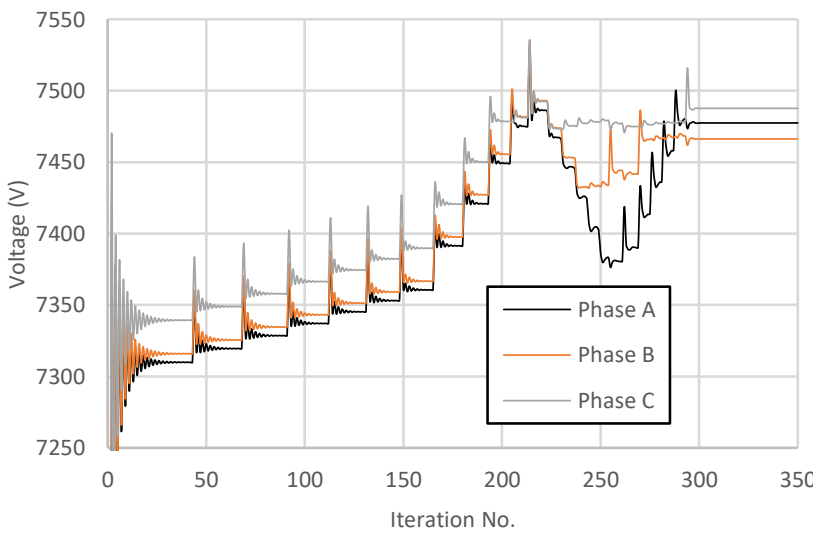

Fig 12. Voltage of SVR 3 throughout the iterative power flow process.

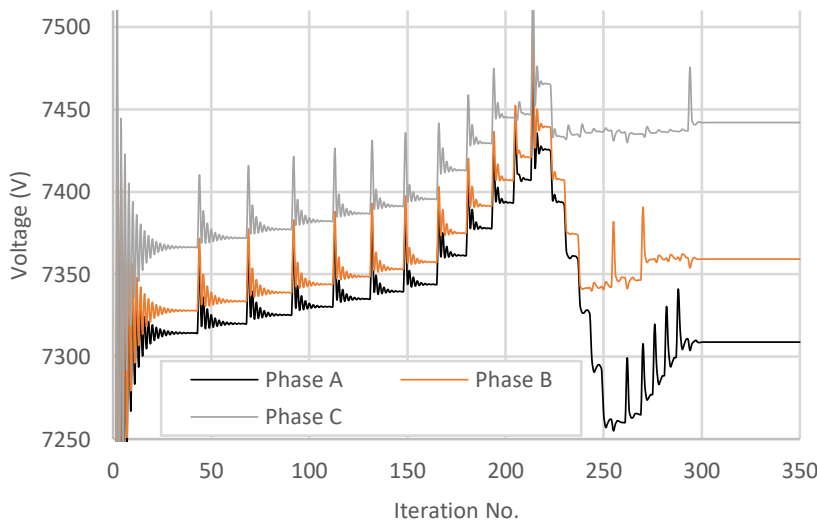

Fig 13. Voltage of capacitor 4 throughout the iterative power flow process

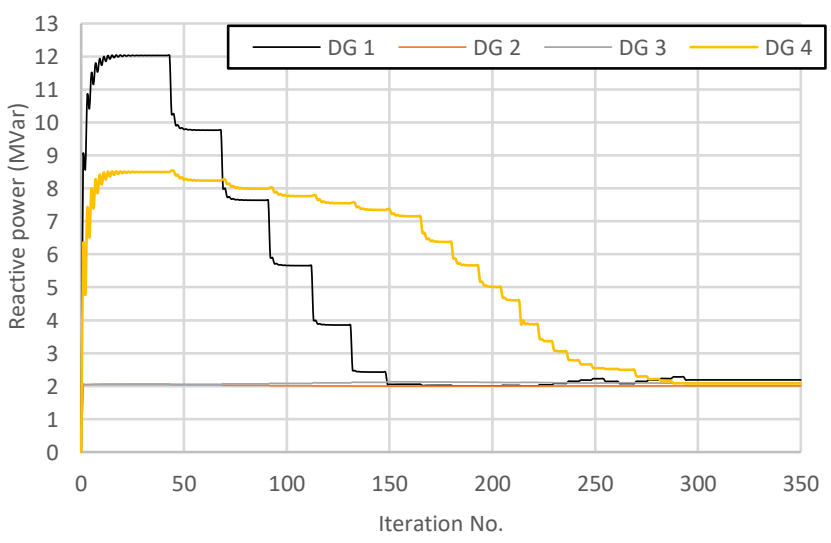

Fig 14. Reactive power of DGs throughout the iterative power flow process. 


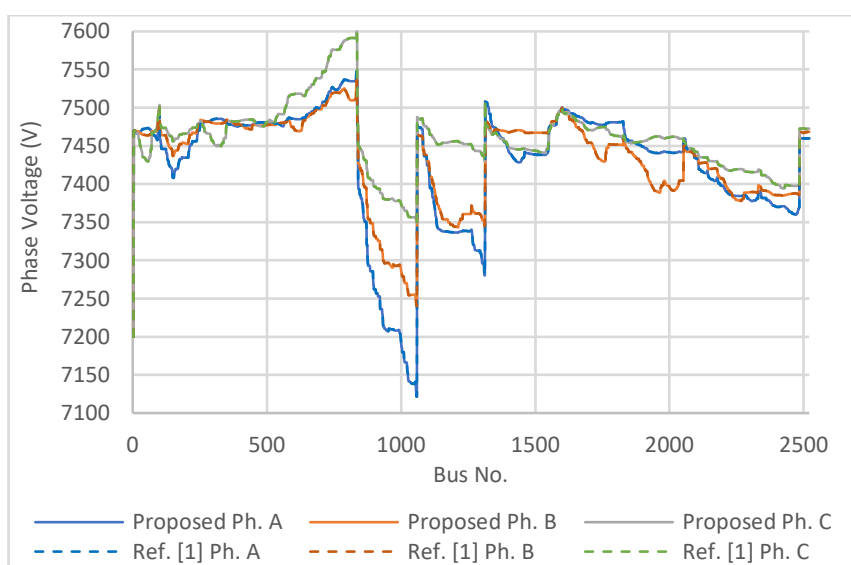

Fig 15. Voltage Profile of the network after all switching actions have been executed. The proposed model and the model of ref. [1] is applied for the simulation of SVRs.

\section{Table 14}

Total computation time of the power flow using the proposed and the model of ref. [1] for the simulation of SVRs.

\begin{tabular}{|c|c|c|}
\hline $\begin{array}{c}\text { Computation time of power flow } \\
\text { iterations }\end{array}$ & $\begin{array}{c}154.5 \mathrm{~s} \\
(309 \times 0.5 \mathrm{~s})\end{array}$ & $\begin{array}{c}154.5 \mathrm{~s} \\
(309 \times 0.5 \mathrm{~s})\end{array}$ \\
\hline $\begin{array}{c}\text { Computation time of the inversion of the } \\
\boldsymbol{Y}_{\boldsymbol{B} U \mathrm{~S}} \text { after each SVR tap variation }\end{array}$ & $0 \mathrm{~s}$ & $\begin{array}{c}1254 \mathrm{~s} \\
(19 \times 66 \mathrm{~s})\end{array}$ \\
\hline Total computation time & $154.5 \mathrm{~s}$ & $1408.5 \mathrm{~s}$ \\
\hline \multicolumn{2}{|c}{} \\
\hline
\end{tabular}

\section{Conclusion}

This paper presents a novel 3-Bus equivalent circuit for modeling three-phase SVRs in the $\mathrm{Z}_{\mathrm{Bus}}$ power flow. The model is applicable in all SVR configurations. Contrarily to the conventional SVR models that include the tap variables inside the $\mathrm{Y}_{\mathrm{BUS}}$ matrix, the proposed model simulates them in the form of current sources, outside the $\boldsymbol{Y}_{\boldsymbol{B} \text { BS }}$ matrix, avoiding its re-factorization or inversion after each tap change. Furthermore, possible convergence issues caused by the low impedance of step voltage regulators are addressed by introducing fictitious impedances, without, however, affecting the accuracy of the model.

The proposed model has been validated against wellestablished commercial softwares such as Simulink and OpenDSS confirming its high accuracy. Furthermore, it has been compared against a recently published conventional SVR model. Simulations results in the IEEE 8500-node network indicate that a time reduction around 1 minute for each tap variation is achieved from the proposed SVR model compared with the conventional one.

To summarize, the proposed SVR model combines three important characteristics: a) increased accuracy since it considers the precise three-phase SVR circuit without any simplification, b) generic applicability since it can be applied in all SVR types and configurations, c) reduced computation time. Therefore, it can be applied to accelerate the execution of several real-time DMS applications such as state estimation, OPF, VVC, OFR, voltage stability, heuristic optimization, which require the subsequent tap variation of SVRs.

\section{Acknowledgement}

«This research is co-financed by Greece and the European Union (European Social Fund- ESF) through the Operational Programme «Human Resources Development, Education and Lifelong Learning» in the context of the project "Strengthening Human Resources Research Potential via Doctorate Research - 2nd Cycle" (MIS-5000432), implemented by the State Scholarships Foundation (IKY).».

The authors are with the School of Electrical and Computer Engineering, Aristotle University of Thessaloniki, Thessaloniki, GR 54124, Greece (email: bobodakis@hotmail.com; kryonidi@ece.auth.gr; minalex@auth.gr).

\section{References}

[1] M. Bazrafshan and N. Gatsis, "Comprehensive Modeling of ThreePhase Distribution Systems via the Bus Admittance Matrix", IEEE Trans. Power Syst., vol. 33, no. 2, pp. 2015-2029, 2018. https://doi.org/10.1109/TPWRS.2017.2728618

[2] W. H. Kersting, "Distribution Feeder Voltage Regulation Control", IEEE Transactions on Industry Applications, Vol. 46, No. 2, pp. 620 $626,2010$. https://doi.org/10.1109/TIA.2010.2040060

[3] C. G. Moránet. al., "4-Node Test Feeder with Step Voltage Regulators", International Journal of Electrical Power \& Energy Systems Vol. 94, pp. $245-255,2018$ https://doi.org/10.1016/j.ijepes.2017.06.027

[4] Baki Cetindag, Ilhan Kocar, Assane Gueye \& Ulas Karaagac, "Modeling of Step Voltage Regulators in Multiphase Load Flow Solution of Distribution Systems Using Newton's Method and Augmented Nodal Analysis", Electric Power Components and Systems, 45:15, 1667-1677, DOI: 10.1080/15325008.2017.1363322, 2017

[5] E.E Pompodakis, G.C Kryonidis, M. C Alexiadis, "A comprehensive load flow approach for grid-connected and islanded AC microgrids", Trans. Power Systems, vol. 35, no. 2, pp. 1143-1155, 2020. https://doi.org/10.1109/TPWRS.2019.2945011

[6] E. E Pompodakis, G. Kryonidis, C. Demoulias, M. Alexiadis, "A Generic Power Flow Algorithm for Unbalanced Islanded Hybrid AC/DC Microgrids", IEEE Transactions on Power Systems (Early Access), July 2020 https://doi.org/10.1109/TPWRS.2020.3012815

[7] I. Džafic, R. A. Jabr, E. Halilovic, and B. C. Pal, "A sensitivity approach to model local voltage controllers in distribution networks," IEEE Trans. Power Syst., vol. 29, no. 3, pp. 1419-1428, May 2014. https://doi.org/10.1109/TPWRS.2013.2290813

[8] Gengwu Zhang, Chengmin Wang, Haibing Wang, Ning Xie, "On the convergence of the implicit Z-Bus power flow method for distribution systems," Electric Power Systems Research, Volume 171, Pages 74 84,2019 https://doi.org/10.1016/j.epsr.2019.02.002

[9] E. Pompodakis, A. Ahmed, M. Alexiadis, "A Three-Phase WeatherDependent Power Flow Approach for 4-Wire Multi-Grounded Unbalanced Microgrids with Bare Overhead Conductors", IEEE Transactions on Power Systems (Early Access), October 2020. https://doi.org/10.1109/TPWRS.2020.3029053

[10] R. Yan et. al, "Modeling and Analysis of Open-Delta Step Voltage Regulators for Unbalanced Distribution Network With Photovoltaic Power Generation", IEEE Trans. on Smart Grid, Vol. 9, no. 3, pp. 2224 - 2234, 2018. https://doi.org/10.1109/TSG.2016.2609440

[11] R. A. Jabr, I. Džafić and S. karaki, "Tracking transformer tap position in real-time distribution network power flow applications", IEEE Trans. on Smart Grid, vol. 9, no. 4, pp. 2442-2452, 2018 https://doi.org/10.1109/TSG.2016.2612177

[12] I. Huseinagić, I. Džafić, R. A. Jabr, "A compensation technique for unsymmetrical three-phase power flow", Int. Symposium. Ind. Electron. (INDEL), Bosnia, 3-5 November, 2016. https://doi.org/10.1109/INDEL.2016.7797812

[13] H-T Neisius, et. al, "Modeling of auto-transformers for load flow calculations", $3^{\text {rd }}$ IEEE PES ISGT Conference Europe, pp. 16, 2012. https://doi.org/10.1109/ISGTEurope.2012.6465850 
[14] I. Dzafic, H.T. Neisius and D. Ablakovic, "Multi process real-time network applications in Distribution Management System," IPEC 2010 Conference Proceedings, vol., no., pp.340-345, 27-29 Oct. 2010 https://doi.org/10.1109/IPECON.2010.5697155

[15] Yukon energy automation software platform, "Integrated Volt/VAR Control." $\quad$ Sep. $2017 . \quad$ [Online]. Available: https://www.eaton.com/content/dam/eaton/products/utility-and-gridsolutions/grid-automation-systems/volt-var-management/volt-varmanagement-software/integrated-volt-var-control-br910005en.pdf

[16] A. Ulinuha et al., "Optimal Scheduling of LTC and Shunt Capacitors in Large Distorted Distribution Systems using Evolutionary-Based Algorithms", IEEE Transactions on Power Delivery, vol. 23, No. 1, pp. 434-441, 2008 https://doi.org/10.1109/TPWRD.2007.911166

[17] IEEE PES. (2020, May), "IEEE PES AMPS DSAS Test Feeder Working Group", [Online]. Available: https://site.ieee.org/pestestfeeders/resources/

[18] Pompodakis, Evangelos; Ahmed, Arif; Alexiadis, Minas (2021): A Sensitivity-Based Three-Phase Weather-Dependent Power Flow Algorithm for Networks with Local Controllers-Part I: Algorithm Development. TechRxiv. Preprint. https://doi.org/10.36227/techrxiv.13489131.v1

[19] Pompodakis, Evangelos; Ahmed, Arif; Alexiadis, Minas (2020): A Sensitivity-Based Three-Phase Weather-Dependent Power Flow Approach for Networks with Local Controllers-Part II: Case Studies. TechRxiv. Preprint. https://doi.org/10.36227/techrxiv.13489140.v1

[20] M. Aryanezhad, "Management and coordination of LTC, SVR, shunt capacitor and energy storage with high PV penetration in power distribution system for voltage regulation and power loss minimization", International Journal of Electrical Power \& Energy Systems, Vol. 100, Pages 178-192, 2018.

[21] R. A. Jabr and I. Dzafic, "Sensitivity-based discrete coordinate-descent for Volt/VAr control in distribution networks," IEEE Trans. Power Syst., vol. 31, no. 6, pp. 4670-4678, Nov. 2016.

[22] Ruiz-Rodriguez, F.J., Hernandez, J.C., Jurado, F., "Iterative harmonic load flow by using the point-estimate method and complex affine arithmetic for radial distribution systems with photovoltaic uncertainties", International Journal of Electrical Power \& Energy Systems 118, 105765, 2020.

[23] J.C.Hernandez, F.J.Ruiz-Rodriguez, F.Jurado, F.Sanchez-Sutil, "Tracing harmonic distortion and voltage unbalance in secondary radial distribution networks with photovoltaic uncertainties by an iterative multiphase harmonic load flow", Electric Power Systems Research 185, 106342, 1, 2020.

[24] I Roytelman et al., "Coordinated local and centralized control in distribution management systems", IEEE Tans Power Delivery, Vol. 15, No. 2, pp. 718-724, 2000

[25] IEEE Standard for Interconnection and Interoperability of Distributed Energy Resources with Associated Electric Power Systems Interfaces, IEEE Std 1547-2018, Feb. 2018.

[26] Kraiczy et. al., "Parallel Operation of Transformers with on Load Tap Changer and Photovoltaic Systems with Reactive Power Control", IEEE Trans. Smart Grid, Vol. 9, No. 6, pp. 6419-6428, 2018.

[27] F. A. Viawan and D. Karlsson, "Voltage and reactive power control in systems with synchronous machine-based distributed generation," IEEE Trans. Power Del., vol. 23, no. 2, pp. 1079-1087, Apr. 2008.

[28] A. Joseph et. al., "Secure High DER Penetration Power Distribution via Autonomously Coordinated Volt/VAR Control", IEEE Transactions on Power Delivery, Vol. 35, No 5, 2020.

[29] D. Ranamuka et. al., "Examining the interactions between DG units and voltage regulating devices for effective voltage control in distribution systems", IEEE Transactions on Industry Applications, Vol. 33, No. 2, pp. 1485-1496, 2017.

[30] D. Ranamuka et. Al., "Online voltage control in distribution systems with multiple voltage regulating devices," IEEE Trans. Sustain. Energy, Vol. 5, No. 2, pp. 617-628, 2014.

[31] Don Wareham, "Step Voltage Regulators", Cooper Power Systems by Eaton, 2013, Accessed on: November 1, 2020. [Online].Available: http://www.cscos.com/wp-content/uploads/NY1839-EatonRegulators-D.Wareham.pdf

[32] Eaton Cooper Power Series, "Voltage Regulators vs. Load Tap Changers", Voltage Regulators Reference Data TD225012EN, 2017.1

[33] A. Bettanin et al., "Voltage management strategies for low voltage networks supplied through phase-decoupled on-load-tap-changer transformers", AEIT Int. Annual Conf. Infrastructures Energy ICT Oppor. Foster. Innov. AEIT 2017, pp. 1-6, 2017.
[34] Fundamentals Ltd Power Systems Technology, "Enhanced Voltage Control EAVC Settings Calculation Guide", Fundamentals Reference F9183, 15/08/2018, [Online]. Available:

https://www.enwl.co.uk/globalassets/innovation/enwl011/enw1011closedown-appendix-d.pdf

[35] I. Roytelman and V. Ganesan, "Modeling of local controllers in distribution network applications," IEEE Trans. Power Del., vol. 15 no. 4 , pp. $1232-1237$, Oct. 2000. 